\title{
Increasing spatial resolution of wind resource prediction using NWP and RANS simulation
}

\author{
Alessio Castorrinia ${ }^{\mathrm{a}, *}$, Sabrina Gentile ${ }^{\mathrm{b}, \mathrm{c}}$, Edoardo Geraldi ${ }^{\mathrm{b}}$, Aldo Bonfiglioli ${ }^{\mathrm{a}}$ \\ ${ }^{a}$ School of Engineering, Università della Basilicata, \\ Viale dell'Ateneo Lucano 10, 85100 Potenza, Italy \\ ${ }^{b}$ CNR Italian National Research Council - IMAA \\ C.da S.Loja - 85020 Tito Scalo (PZ), Italy \\ ${ }^{c}$ Center of Excellence Telesensing of Environment and Model Prediction of Severe events (CETEMPS), \\ University of L'Aquila, 67100 L'Aquila, Italy
}

\begin{abstract}
The detailed prediction of the upcoming wind on wind farms can support optimization of wind energy production and operation and maintenance. Numerical Weather Prediction (NWP) tools allow to simulate the wind over long-term forecasting horizons (up to several days) with a spatial resolution ranging between the continental level down to a few hundred meters. We present a methodology, based upon Computational Fluid Dynamics (CFD) and Reynolds Averaged Navier Stokes (RANS) modelling, that allows to downscale the spatial resolution of the wind prediction supplied by a NWP model down to the typical length-scale of wind energy applications. The proposed approach combines a number of standard tools, including: Geographical Information Systems (GIS), Advanced Research - Weather Research and Forecasting (WRF-ARW) and OpenFOAM, and proposes methods to interface these tools and set-up the localscale simulation. Models and problem sizes are selected to keep the computational cost of the system sustainable in view of its implementation in operational forecasting. Finally, we present the application of the method on a given onshore site, and for three different meteorological conditions, showing the potential of the approach, but also giving an account of the limitations that it may encounter when dealing with complex planetary boundary layers.
\end{abstract}

Keywords: wind, mesoscale, RANS, WRF, NWP

${ }^{*}$ Corresponding author. e-mail: alessio.castorrini@unibas.it 


\section{Introduction}

Weather forecast and aerodynamic simulations represent powerful tools for wind energy prediction. The capability of accurately predicting the incoming wind flow, can be of great help in optimizing the power extraction from a wind farm, the power management in the distribution and, eventually, accumulation systems, but also in the management of the parking time, which is necessary for the ordinary maintenance of the wind farm.

In order to be able to accurately predict the wind over a specific wind power generation site, we need to encompass multiple phenomena, each being characterized by its own temporal and spatial scales. Multiscale approaches help to segregate the solution (simulation) process, starting from the larger scales and progressively refining toward the smaller scales. In the case of wind prediction we need to separate the phenomenon on three main levels: planetary scale, mesoscale and local scale. For each of these scales we need to adopt a specific and dedicated modelling. It is possible to consider feedback effects in order to obtain a fully coupled system (closed loop), or to make the hypothesis that the influence of the smaller scales on the larger ones is negligible, when compared with other embedded sources of error.

The weather forecast simulation tools represent a mature technology that is able to predict, several hours in advance, the behaviour of the atmospheric wind at the mesoscale level. The mesoscale models are numerical weather prediction (NWP) systems able to simulate the dynamics of the atmosphere, solving the nonlinear equations of motion and continuity equations for mass, heat and water vapour. The model physics related to boundary layer, surface, clouds, radiation and convection is represented through sophisticated parameterizations. The Advanced Research Weather Research and Forecasting (WRF-ARW), the NWP used in this study, it is very flexible and suitable to a wide range of meteorological events across scales from hundreds of meters to thousands of kilometers. WRF-ARW supports progressive nesting (one- or two-way) to refine the solution and to allow a spatial resolution enhancement through additional domains [1; 2]. 
Wind farms, however, have a characteristic length scale of the order of meters, so that the use of a mesoscale simulation tool to reach this level of spatial resolution would require massive computational resources. Furthermore, the mathematical models available in the mesoscale simulation tools are not suitable for the accurate characterization of the local, three-dimensional effects that affect the flow-field at the wind turbine level.

Computational Fluid Dynamics (CFD) methods for the simulation of three-dimensional turbulent flows are largely adopted to study aerodynamic flows characterized by length scales much closer to wind turbine applications. We can find CFD applications for wind energy ranging from the airfoil scale [3], to the full blade [4], and wind farm wake studies [5]. In the present study, the CFD simulations will be performed using the Open-source Field Operation and Manipulation (OpenFOAM) [6] toolbox.

We present here a multiscale methodology that allows to easily integrate the WRFARW and CFD simulation for complex turbulent flows in order to produce spatially refined hourly predictions of the wind resource, available in selected areas of the order of a few kilometres squared. The tool could be integrated as an add-on of the weather forecast simulation platform to zoom and increase the spatial resolution of the prediction within for specific areas of interest.

One of the novelties of the present study is the proposal of a method to couple these two predictive tools, making the downscaling not an offline post-processing of past data, but a method to increase the spatial resolution of the forecasting, that can be used in operational mode. Furthermore, we test the capability of two different state-ofthe-art RANS models (accounting, or not, for buoyancy effects) to deal with complex stratified atmospheric boundary layers. Finally, we emphasize the importance of using opensource software and provide details of the interfaces that we developed to couple the two simulation tools, so that the proposed forecasting framework can be easily reproduced.

A large spectrum of applications involving the coupling between NWP and CFD models can be found in the field of urban flow simulation and pollutant transport, see e.g. [7, 8, 9]. Recent studies dealing with the accuracy of the coupling between mesoscale simulation codes and CFD codes are available. Temel et al.[10] compare the CFD solution of the Planetary Boundary Layer (PBL) on reference benchmarks 
obtained using the theoretical inflow wind profile (based on the Monin-Obukhov Similarity) with that obtained by prescribing the inflow supplied by a mesoscale simulation, showing the possibility to attain good predictions also on complex terrain. Tewari et al. [11] studied the impact of coupling a CFD model with a mesoscale model on urban contaminant transport, reporting on a quasi-steady coupling approach at the boundary. Recently, Duran et al. presented in [12] a similar down-scaling framework for wind resource assessment, which also makes use of NWP and RANS simulations. Their work is focusses on the use of the meso-microscale coupling to estimate the annual wind features in selected areas. The mesoscale prediction is averaged over a year, for a set of angular intervals covering all possible wind directions and for each of these wind directions, the microscale model is used to downscale the prediction. In [12], the authors compare the results with anemometric data showing good agreement of the prediction, with some limitations for stably stratified boundary layers. This latter aspect is also arising in the present study. Other examples of mesoscale - microscale simulation coupling can be found in $[13,14,7,8,15]$.

Referring to the wind energy application, an important impact is given by the research done in the framework of the Windbench European project [16]. Benchmark research on the CFD modeling that is best suited to simulate planetary boundary layers with Reynolds-averaged Navier Stokes (RANS) models has been driven by Richards and Hoxey [17] and Parente et al. [18]. Blocken et al. [19] report the validation against field measurement in Ria de Ferrol (Spain), showing that the computed averaged wind falls within $10 \%$ - 20\% percent of the measurements. Balogh et al. [20] compare different implementations of the RANS models proposed in [18], verifying its accuracy over not flat terrains. RANS simulations are also often adopted to simulate the interaction between wind farms, PBL and complex terrains [21, 22].

Unsteady RANS (URANS), Detached Eddy Simulation (DES) and Large Eddy Simulation (LES) turbulence modeling have all been tried (see e.g. [23]) in the attempt to increase the spatial and temporal resolution of the mesoscale simulation. Even though each of these turbulence modeling options has its own pros and cons, all of these studies have confirmed that the integration between mesoscale and CFD simulations is indeed possible and offers the possibility to focus and down-scale the wind prediction 
in selected areas of the size of a wind farm.

With the increase of the computing power available to the many players in the wind energy sector, we propose a step forward. We will show that is feasible and reliable to couple the time-marching weather prediction supplied by the WRF-ARW mesoscale model, with the local-scale steady RANS simulation of the wind over a specific area. In doing so, a time averaging strategy will be proposed to reduce the time-dependent mesoscale data to a coarser set of time-averaged snapshots.

The method will then be applied to downscale the wind prediction for different wind conditions during three days between 2019 and 2020. The tests demonstrate the applicability and accuracy of the methodology, but also its critical aspects, which are related to the choice of the averaging strategy and RANS modelling.

We point out that the proposed system makes the forecast available at a spatial resolution compatible with the prediction of the wind at the specific wind turbine location within the wind farm. This information, which is supplied with a step of a few hours (the length of each snapshot), can help the management of the system, paving the way to more sophisticated solutions to control the wind farm in operation.

The paper is organized as follows: Section 2 describes the methodologies and mathematical models adopted for both the mesoscale and local scale simulations, and their mutual interface. The application of the combined methodologies to set-up a reference case study, using the open-source software for NWP (WRF-ARW) and CFD (OpenFOAM), is detailed in Section 3 Section 4 shows the results obtained for the case study in terms of downscaling of wind prediction in a given site (corresponding to a weather measurement station) and for three days selected in three different seasons. For one of these cases, a more detailed study is presented about the effect of adopting two different RANS models. The steady k- $\varepsilon$ RANS with and without the Boussinesq approximation [24] and temperature equation, are applied to a case featuring a stratified wind profile which exhibits a strong variations of velocity and direction along the altitude. Two supporting Appendixes report i) the validation of the local scale PBL RANS modelling on the Askervein's Hill benchmark case, and ii) a more detailed description of the algorithm used to apply the heterogeneous land coverage boundary condition in OpenFOAM. 


\section{Methodology}

Many aspects, models and techniques are involved in the process. For each of these we shall hereafter present a brief overview by defining the problem and the technical solution adopted.

Once the target site has been defined, the local-scale wind field prediction will be affected by:

- The orographic detail of the area under scrutiny;

- The land-use in terms of roughness length distribution, and presence of obstacles (forests, buildings);

- The space-time prediction of the wind at the mesoscale level and the way the data are transferred from mesoscale to local-scale;

- The turbulence modelling adopted for the local-scale simulation.

The local scale simulation aims at increasing the spatial resolution of the mesoscale prediction. However, the wind is a stochastic phenomenon which not only varies in space, but also in time. To properly account for the time variation, one should directly couple the dynamic, i.e. time-accurate, solution of the mesoscale model, with a timeaccurate CFD simulation. Several examples of this strategy have been proposed in the literature, see e.g. [23], but they are obviously characterized by a significant computational cost. Rodrigues et al. [14] coupled the WRF mesoscale solver [1] with an URANS CFD solver to estimate the local turbulence intensity and wind shear in different periods of the day. An example of coupling between unsteady mesoscale simulation and LES local-scale simulation can be found in [15], where an immersed boundary LES approach is coupled with WRF to estimate the fine-scale urban dispersion.

Our choice consists in synchronizing mesoscale and localscale simulations so as to obtain a spatially refined prediction of the wind in one or multiple selected areas. Such a synchronization must be computationally affordable in order to preserve the capability of the system to deliver wind forecasts at a computational cost of the order of a few hours. Clearly, the dynamic (or time-accurate) coupling of the mesoscale model 
with either URANS or LES would not respect this constraint, requiring significant computational resources. The idea here is to take the time history of the wind supplied by the mesoscale model and perform an automatic average over specific time-intervals. This averaging procedure produces a sequence of snapshots of the wind evolution for the selected interval of forecast. For each snapshot we then perform a steady RANS calculation that allows us to increase the spatial resolution of the wind prediction during the selected time interval.

\subsection{Mesoscale model}

The NWP tool used to simulate the mesoscale wind is the WRF-ARW model, a next-generation NWP system designed for flexible purposes ranging from operational forecasting applications to specific atmospheric studies. The WRF-ARW dynamical core system has been developed by the collaborations of several research institutes guided by the National Center for Atmospheric Research (NCAR, http: //www.wrf-model.org) The WRF-ARW supports horizontal nesting (one or two-way) to allow a horizontal resolution focus over a region of interest by introducing an additional domain into the simulation. The full physics parameterizations (cumulus convection, microphysics, radiation, planetary boundary layer and land-surface) provided by WRF vary from simple and efficient to the most refined and computationally costly [1, 2, 25]. These multi-physics package options allow flexibility in treating the physical processes, so this NWP can be applied to a wide range of applications, ranging from solar irradiance [26, 27, 28, 29, 30] to wind energy prediction [31, 32, 33]. Concerning the wind resource, WRF-ARW is mainly used with maximum horizontal resolutions of the order of a few kilometers (3-5 km) which is not sufficient to accurately reproduce this resource on a specific wind power generation site [31, 32, 33]. In only few works [34, 35], WRF-ARW is used in LES mode, reaching horizontal resolutions of the order of a hundred meters, but these simulations have a high computational cost and therefore cannot be used for operational purposes.

\subsection{RANS modelling for local scale simulation}

The RANS modelling adopted for the present application is the k- $\varepsilon$ model in its adaptation for PBL reported in [36], and based on the formulation proposed by Richards 
and Hoxey [17]. The model has been formulated to preserve the PBL profile on an empty domain, with constant surface roughness, and it is recognized as one of the most used turbulence models for simulating the PBL with CFD solvers.

Starting from the standard k- $\varepsilon$ model [37], the PBL formulation is obtained by imposing the consistency between the $\mathrm{k}-\varepsilon$ transport equations, written for an homogeneous 2D PBL, and the following profile law:

$$
\begin{aligned}
u & =u_{*} \ln \left(\frac{z-z_{0}}{z_{0}}\right) \\
k & =\frac{u_{*}^{2}}{\sqrt{C_{\mu}}} \\
\varepsilon & =\frac{u_{*}^{3}}{\kappa\left(z+z_{0}\right)}
\end{aligned}
$$

The homogeneous 2D PBL formulation is obtained by assuming a zero vertical velocity, constant pressure and constant shear stress $\tau_{0}=\rho u_{*}^{2}$. With these assumptions the transport equations reduce to:

$$
\begin{array}{r}
\frac{\partial}{\partial z}\left(\frac{\mu_{t}}{\sigma_{k}} \frac{\partial k}{\partial z}\right)+G_{k} \frac{\varepsilon}{k}-\rho \varepsilon=0 \\
\frac{\partial}{\partial z}\left(\frac{\mu_{t}}{\sigma_{\varepsilon}} \frac{\partial \varepsilon}{\partial z}\right)+C_{\varepsilon 1} G_{k} \frac{\varepsilon}{k}-C_{\varepsilon 2} \rho \frac{\varepsilon^{2}}{k}=0
\end{array}
$$

where $G_{k}=\mu_{t}\left(\frac{\partial u}{\partial z}\right)^{2}$ and $\mu_{t}=\rho C_{\mu} \frac{k^{2}}{\varepsilon}$. Following [36] and [17], the model constants are set as follows: $\kappa=0.4, \sigma_{k}=1, \sigma_{\varepsilon}=1.11, C_{\mu}=0.09, C_{\varepsilon 1}=1.44$ and $C_{\varepsilon 2}=1.92$.

We notice that the model above works well for the given theoretical inflow velocity profile (1), as shown in the validation test in Appendix 1 and, e.g. in [17; 10].

However, we expect, and verified, see Sect. 3, that the inflow boundary condition supplied by the mesoscale simulation does not necessarily match the theoretical velocity profile (1). In particular, whenever the ground and the geostrophic currents are oriented differently, it is possible to encounter a wind profile characterized by different layers and significant changes in wind direction along the height. The study of this latter case is more difficult to find in the literature. Alinot et al. [38] propose to introduce the buoyancy terms in the RANS modelling, by adopting the Boussinesq approximation [24] to account for the density variations among the various atmospheric layers. Following this same approach, we will also conduct a second set of simulations 
in which the gravity source term is accounted for in the momentum equation and the density of the fluid (which appears in the gravity term) varies linearly with temperature. When using the aforementioned approach, the temperature (energy) equation is also added to the governing PDEs.

\subsection{Time averaging strategy}

Since the prediction reliability of the mesoscale model decreases progressively after 24 - 48 hours of forecast from the start time accordingly with the synoptic conditions [39], an interval $T$, corresponding to the first 12 hours of simulation, has been selected. A finite set of snapshots (or time-averages) has been extracted from the time history of the wind speed obtained from the mesoscale simulation over the time frame $T$. Each of these shapshots supplies the steady boundary conditions required by the RANS simulations. The number of snapshots should be limited, so as to keep the computational cost within affordable limits, but also sufficient to adequately describe the time variation of the wind over $T$. Using the same logic adopted in Welch's method [40], we propose the following overlapping, evenly-spaced method to define the snapshots. The time interval $T$ is split into $\mathrm{N}$ data segments of length $\mathrm{M}$, overlapping by $\mathrm{D}$ points. A value of $\mathrm{D}=\mathrm{M} / 2$ corresponds to a $50 \%$ overlap and a value of $\mathrm{D}=0$ to no-overlap. The value of the velocity components obtained from the mesoscale simulation is then averaged on each time segment.

\subsection{Definition of RANS domain and boundary conditions from mesoscale results}

\subsubsection{Domain definition}

We use the terrain and mesoscale grid to define the boundaries of the local-scale computational domain, i.e. the domain where the RANS solution is computed. The choice of the size of the CFD domain has to reconcile two competing requirements. On the one hand, in order to describe the wind with an adequate level of accuracy, we have to simulate an area that is large enough to guarantee that the border effects due to the direct imposition of the mesoscale solution along the boundaries of the CFD domain, affects the target site. On the other hand, the selection of a too large area for the CFD domain would force us to increase the mesh spacing (so as to keep the 
computational costs under acceptable levels), thus reducing the spatial resolution of the local scale simulation.

The ground face of the computational domain is defined by the digital model of the terrain. Concerning the top boundary, we use an horizontal plane whose elevation is given by the largest value of the PBL height (PBLH) predicted by the mesoscale model in the selected area.

\subsubsection{Wind boundary conditions}

As reported by Hargreaves and Wright [36], the PBL is driven by the geostrophic wind. In the CFD modelling, energy is removed because of the shear stress at the ground and the geostrophic winds are "felt" only close to the top boundary patch. Therefore, the simple application of a wind profile along the inflow boundary patch cannot guarantee the correct transport of the PBL throughout the entire fluid domain.

To mitigate this effect we impose the wind velocity profile obtained from the mesoscale simulation on all the boundary patches, including the top surface. By doing so, we prescribe inflow and outflow winds that are consistent with the mesoscale wind field, thus enforcing convection also at the top boundary patch.

From a practical point-of-view, we need to transfer the wind velocity components from the mesoscale solution to the boundaries of the local scale computational domain. For operational forecasting, the output of the mesoscale solution is not saved for all the time steps (typical writing frequency is one per hour), in order to save memory and performance thus extending the forecast horizon. To apply the averaging method described in Sect. 2.3, however, we have to save the mesoscale solution at each time step. This is not a major issue in terms of storage, because the entire CFD domain (see Fig. 8 of Section 3.4) spans only a few cells of the mesoscale, finer-level grid. Therefore, the mesoscale solution needs to be stored at each timestep only within a limited number of (mesoscale) grid-points, thus making the procedure feasible.

The list of the CFD grid-points where data-transfer from the mesoscale grid is needed is the following:

- four points at the four corners of the local scale computational domain; 
- an additional number of points along each lateral face, which correspond to the intersections between the grid-lines of the mesoscale grid and the lateral patches of the CFD grid;

- one or more mesoscale grid points falling inside the local scale domain.

For each of these solution points, the mesoscale solver saves the time history of the wind velocity components for all the elevation layers inside the PBL, and the PBLH. Each of these fields is then averaged following the strategy outlined in Sect. 2.3. A bilinear interpolation scheme is used to obtain the averaged values over the boundary surfaces of the local scale domain. Zero velocity is imposed on the ground edges of the boundary sides.

A slight unbalancing of the mass flow rate across the CFD domain boundaries is generally observed, because of the higher resolution of the ground model in the local scale simulation. Let us call $\Delta G$ the surface integral of the mass flow rate over all the CFD domain boundaries. If $\Delta G \neq 0$, we apply the following procedure to guarantee mass conservation along the CFD domain boundaries: i) add $\Delta G / 2$ to all the inflow boundary patches; ii) subtract $\Delta G / 2$ to all the outflow boundary patches; iii) iterate if needed. The correction is given in the form of a uniformly distributed velocity in proportion to the flow rate unbalance.

\subsubsection{Ground boundary conditions (land use)}

A wall function based on the boundary layer model described by Equation (1a) [17] is adopted to evaluate the wind velocity profile and turbulence quantities at the ground. In the implementation of the proper ground boundary condition we must account for the land use, in terms of characteristic roughness length, consistently with the parameter $z_{0}$ of Equations 11 .

The digital information about the land use in Europe can be found in the framework of the European Commission program Corine Land Cover (CLC) [41]. In this case, the digital model associates a numerical code, specific to the particular kind of terrain covering present in the map points. As reported by Silva et al. in [42], it is possible to associate different values of the characteristic roughness lenght to each code. 

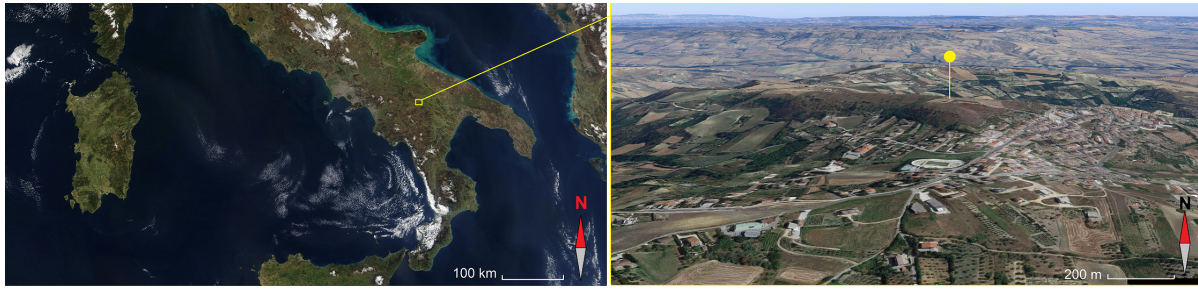

(a) Site location (left) and surrounding land view (right). Source: https://www.google.com/maps/

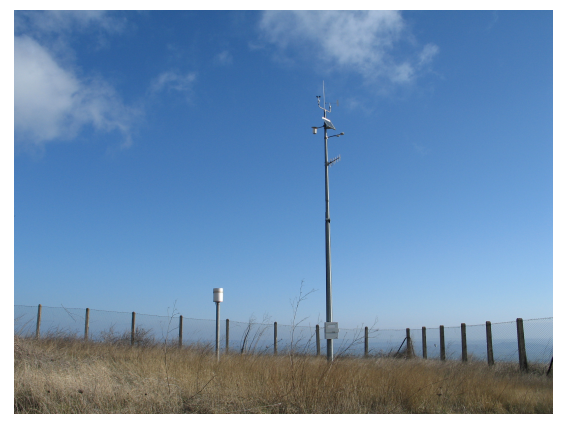

(b) Measurement station.

Figure 1: Site location and measurement station (yellow mark).

\section{Application}

In this section we present an application of the method to a real site. The prediction of the wind field will be done for a site located in the South of Italy, more precisely in $40^{\circ} 45^{\prime} 50^{\prime \prime} \mathrm{N}$ and $15^{\circ} 59^{\prime} 8^{\prime \prime} \mathrm{E}$. The location corresponds to a weather observation station of the "Protezione Civile - Regione Basilicata" 1 the site elevation above sea level is $761 \mathrm{~m}$. The orography of the area, as well as its geographical position, make this site particularly interesting for wind energy production; indeed, several wind farms can be found in the neighbourhood. Fig. 1a shows the location of the measurement station and the surrounding land.

The time periods of the tests correspond to the hours ranging between 8:00 and 20:00 CET of October 19 ${ }^{\text {th }}, 2019$ (day "1"), between 10:00 and 18:00 CET of De-

\footnotetext{
${ }^{1}$ Protezione Civile - Regione Basilicata, Dipartimento Infrastrutture e Mobilità - Corso Garibaldi, 139 - 85100 Potenza,http://www.protezionecivilebasilicata.it/protcivbas/home.jsp
} 
cember 11, 2019 (day "2"), and between 10:00 and 18:00 CET of June 2, 2020 (day "3").

\subsection{Measurements}

Figure $1 \mathrm{~b}$ shows a picture of the measurement station. The device $\mathrm{e}^{2}$ used in the measurement is composed of two elements: a weathercock gonio-anemometer to measure the wind direction and a three-cup tacho-anemometer to measure the wind speed. All measurements are elaborated by a central processing unit and averaged over a period of 15 minutes.

\subsection{Mesoscale simulation}

This study employs the WRF ARW system version 4.0 which is operational since 2016 at the Institute of Methodologies for Environmental Analysis of the National Research Council (IMAA-CNR).

The computational meso-scale grid is characterized by two domains nested in twoway mode. The low-resolution domain $(155 \times 125$ cells $)$ covers the southern part of Italy with a grid spacing of $3.6 \mathrm{~km}$ and it uses as initial and boundary conditions the analysis at 0.125 degrees released by the European Centre for Medium-range Weather Forecasts $($ ECMWF). The nested grid $(403 \times 322$ cells $)$ covers southern Italy and it runs at a resolution of $1.2 \mathrm{~km}$. Fig. 2a shows both the $3.6 \mathrm{~km}$ and $1.2 \mathrm{~km}$ domains. Fig. $2 \mathrm{~b}$ shows a closer view of the area surrounding the measurement station, reporting also the ground elevation. The vertical grid is common to the two domains with 40 vertical levels, ranging from the surface to the upper boundary which is set to 100 $\mathrm{hPa}$. The vertical levels feature an unequally spaced distribution with the first level at approximately $10 \mathrm{~m}$ above the ground and a higher density (the first 15 levels) within the PBL.

Concerning time integration, the time step is set to $20 \mathrm{~s}$ for the largest domain and to $6.67 \mathrm{~s}$ for the finest one.

\footnotetext{
${ }^{2}$ Further information concerning the measurement station can be found at the following link: http: //centrofunzionalebasilicata.it/it/sensoriTempoReale.php?st=VV
} 
The physics options used for the WRF run included the Thompson aerosol-aware microphysics scheme [43], the longwave RRTM radiation scheme [44], the Dudhia shortwave radiation parameterization [45], the Mellor-Yamada Nakanishi and Niino Level 2.5 turbulence closure PBL scheme [46] and the Noah Land Surface Model for the land surface option [47]. Since the grids used in both domains are higly resolved in the horizontal directions, the convective motion is explicitly calculated by the model, so the cumulus parametrization is not necessary.

Fig. 3 shows the weather conditions on October $19^{t h}, 2019$. More precisely, Fig. $3 \mathrm{a}$ (left) shows that a deep cyclonic circulation on the east of Great Britain and a ridge extending on the east Mediterranean basin characterize the synoptic structure of that day. The surface low-pressure system is phased with the higher levels of the atmosphere defining stable conditions for all day. The southern part of Italy is protected by the high mean sea level pressure, guaranteeing clear sky all over the area. This barotropic configuration produces a zonal flow over the Tyrrhenian Sea. Fig. 3a (right) reports the surface wind simulated by the mesoscale model: the area is characterized by a weak wind ranging from 2 to $6 \mathrm{~m} / \mathrm{s}$ with a predominant south-easterly flow; the in-land wind has a variable direction.

For the second case study (December 11, 2019) the geopotential height at $500 \mathrm{hPa}$ shows a double area of low pressure, a deeper one centered at the northern part of Great Britain and the weaker one at the Ionian Sea (Fig. 3b, left). The strong Azores anticyclone expands over the northern and central part of Italy. This marked baric variability leads to intense winds especially in the southern Italian regions. In the area of the measurement station the wind is moderate from North-West (Fig. 3b right).

On the 2nd June 2020 the mean sea level pressure shows a maximum at the North Atlantic Ocean and a trough near Turkey (Fig. 3c, left). The Italian peninsula is in the mid of these two circulations producing a northern ventilation over the area of interest (Fig. 3c, right).

\subsection{Terrain modelling}

To create the surface mesh that is necessary for the definition of the bottom surface of the local scale computational domain, we start from the Digital Terrain Model 
(DTM) file of the Basilicata Region, generated and owned by Regione Basilicata - Centro cartografico dipartimentale della Direzione Generale del Dipartimento Ambiente e Territorio. Detailed information about the DTM used in this application are given in Table 1. while further information about the production of the DTM can be found at [48].

The following procedure is then applied to pass from the DTM file to the Computer Aided Design (CAD) model of the terrain surface:

- Transform the Coordinate Reference System (CRS) from ETRS89/UTM zone $33 \mathrm{~N}$ to Lambert Conformal Conic (LCC) used in the WRF domain;

- Extract a surface of $20 \times 20 \mathrm{~km}$ around the central point given by the measurement station coordinates;

- Project the surface on a Cartesian frame of reference;

- Generate the contour lines of elevation;

- Generate a point-cloud using the contour lines generated in the previous step;

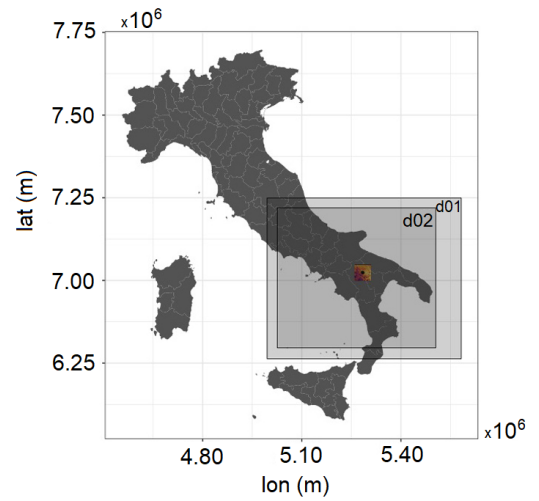

(a) Model domains for the mesoscale simulation: d01 the mother domain with a grid spacing of 3.6 $\mathrm{km}, \mathrm{d} 02$ the inner domain with $1.2 \mathrm{~km}$ horizontal resolution.

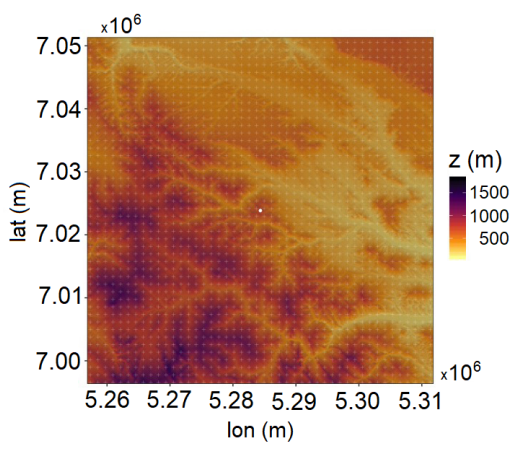

(b) $50 \times 50 \mathrm{~km}$ area of the WRF grid (white vertices), surrounding the measurement station (white circle); colour map of the ground elevation in the background.

Figure 2: Mesoscale grids and ground elevation around the measurement station. 
- Triangulate the surface using the Computational Geometry Algorithms Library (CGAL) [49] advancing front surface reconstruction algorithm to generate a
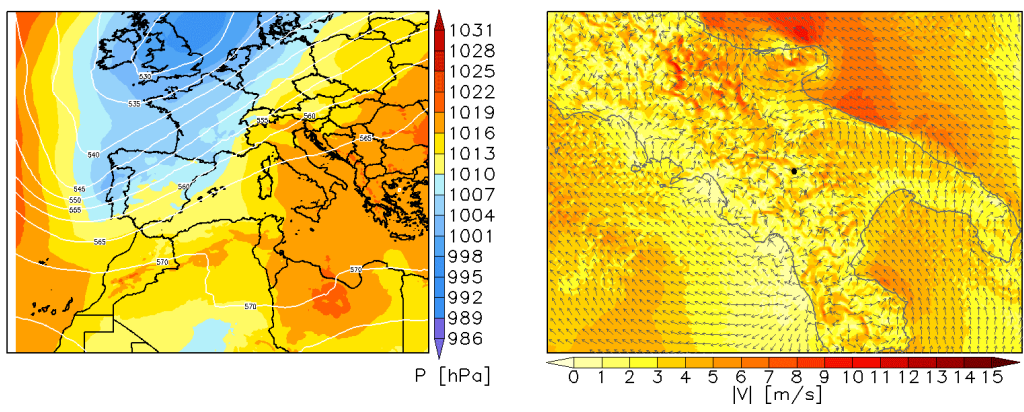

(a) Weather conditions on day "1", October $19^{\text {th }}, 2019$.
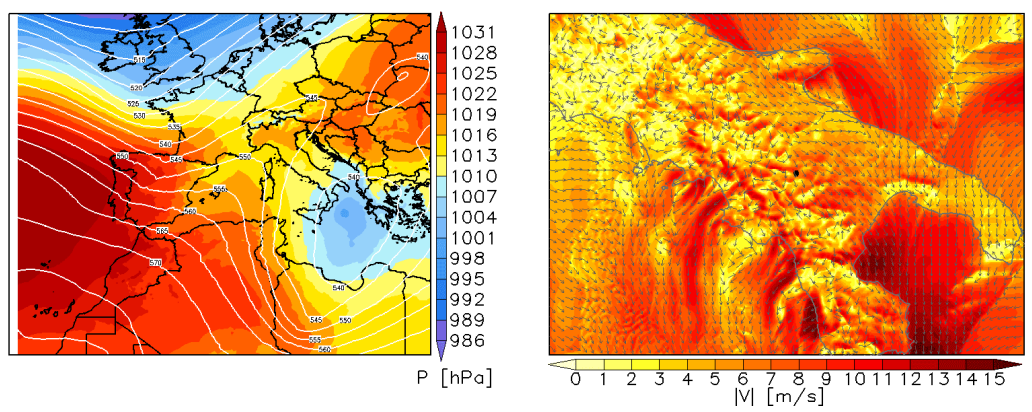

(b) Weather conditions on day "2", December 11 $1^{\text {th }}, 2019$.
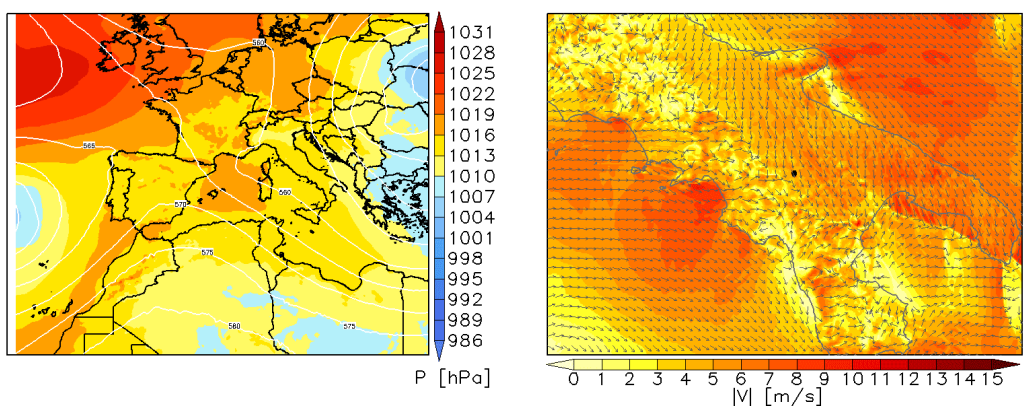

(c) Weather conditions on day "3", June $2^{\text {nd }}, 2020$.

Figure 3: Left: European Centre for Medium-Range Weather Forecasts (ECMWF) 500 hPa geopotential height (white lines) and ECMWF mean sea level pressure at 13 CET. Right: Wind speed and direction at 10 meters simulated by the mesoscale model at $17 \mathrm{CET}$; the black marker indicates the measurement station. 
Year of take-over

DTM Resolution

CRS

Date of publication

Licence

Full extension (WGS84)

Conformity

Output format
2013

$5 \mathrm{~m}$

ETRS89/UTM zone 33N

2016-05-27

IODL 2.0 - link: http://www.dati.gov.it/iodl/2.0

$(39.885,15.331)-(41.143,16.887)$

Regulation (UE) N. 1089/2010

ASCII GRID

Table 1: DTM file details.

closed triangulation of the terrain surface;

- Export the triangulation in STL (Standard Triangulation Language).

Fig. 4 shows a sketch of the main steps of the aforementioned procedure.

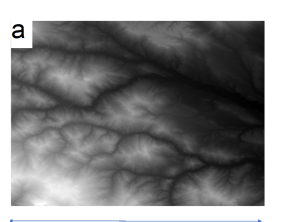

$\sim 0.25^{\circ}$

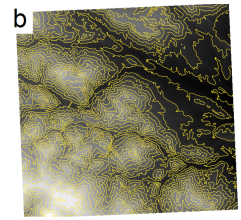

$\sim 20 \mathrm{~km}$
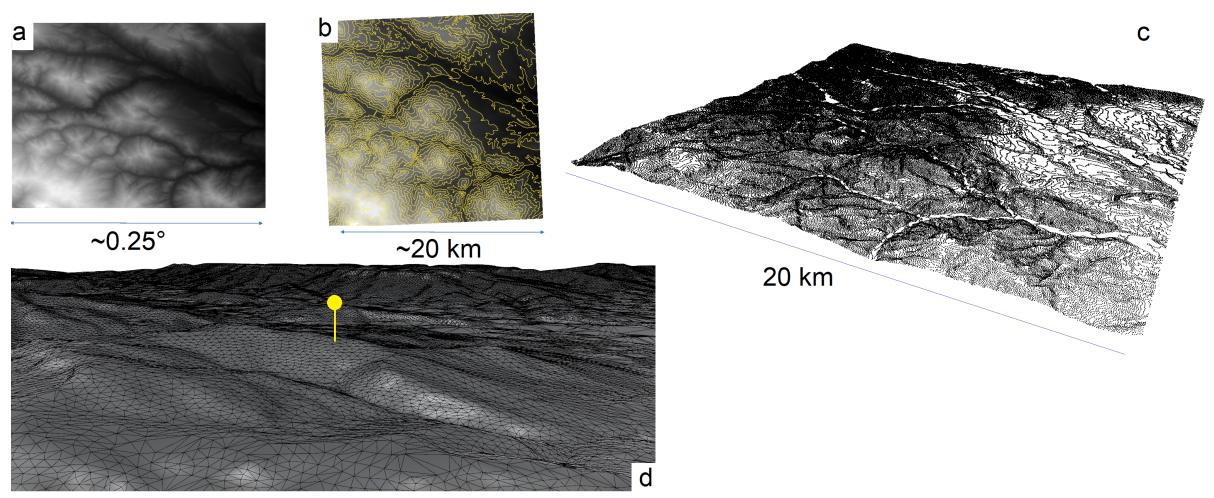

d

Figure 4: Sketch of the procedure used for the terrain model generation. DTM cut in WGS84 (a), DTM cut in LCC with iso-elevation lines (b), point-cloud in Cartesian reference space (c), zoom of the surface triangulation and measurement station location (the yellow pointer) (d). 


\subsection{CFD simulation}

\subsubsection{Computational domain}

We select a squared area, see Fig. 5 and Fig. $6 \mathrm{~b}$, with a side length of abou ${ }^{3} 6 \mathrm{~km}$, centred around the measurement station coordinates. The height of the computational domain is chosen as described in Sect. 2.4.1, i.e. the time-averaged, maximum height of the PBLH predicted by the mesoscale simulation in this area ( $1500 \mathrm{~m}$ for day "1", $\sim 1250 \mathrm{~m}$ for day "2" and $\sim 2350 \mathrm{~m}$ for day "3").

We are free to orient the computational domain arbitrarily, because on all the domain sides and on the top patch we apply the boundary conditions supplied by the mesoscale simulation. As shown in Fig. 55, we choose to align the $x$ axis of the domain with the West to East direction, and the $y$ axis with the South to North direction.

Fig. 6 shows the mesh used for the computations of day "1" (October 19th, 2019): it is a structured mesh with 4.4 million hexahedral cells, 60 points in the $z$ (vertical) direction and 271 along both the $x$ and $y$ directions, which amounts to an average horizontal mesh spacing of about $20 \mathrm{~m}$. The mesh has been further refined within a box of $2 \times 2$ kilometers, centred around the measurement station coordinates. The minimum height of the cells adjacent to the ground is 0.6 meters. Fig. 6a gives a view of the spatial discretization at the ground, while Fig. 6c shows a section of the mesh. The same discretizations in $x$ and $y$, as well as the minimum height and vertical distribution along $z$, have been used to build the computational grids used in the other 2 days of simulation. The reason for building three different grids covering the same area is that the PBLH, which defines the location of the top boundary, is different among the three days.

The difference in vertical discretization between the mesoscale and local scale simulation is shown in Fig. 7 From Figure 5, 6 and 7 we can notice how the downscaling procedure allows to increase the spatial resolution, passing from a set of $4 \times 4 \times 11$ grid-points to a set of around 4 millions of grid points covering the same area.

\footnotetext{
${ }^{3}$ Notice that the WRF output can only be extracted in the WRF grid points; therefore, the CFD domain boundaries have to be adjusted in accordance with the location of the WRF grid points.
} 


\subsubsection{Inflow and outflow boundary conditions}

As explained in Sect. 2, we perform a steady incompressible RANS simulation to increase the spatial resolution in the target area which surrounds the measurement station. This requires to $i$ ) time-average the WRF solution over a pre-set time period, but also to ii) interpolate it on the grid used in the CFD simulation, because the mesoscale has a much coarser resolution (cell size) than the local-scale one: about $1.2 \mathrm{~km}$ vs. $20 \mathrm{~m}$. These two operations are done by post-processing the time-dependent WRF solution at the sampling points defined in Sect.2.4.2 and shown in Fig. 8

The dashed line in Fig. 8 marks the boundary of the local scale computational domain, thus showing how it is nested inside the mesoscale grid: each later face of the CFD domains covers five WRF cells.

As far as the sampling points are concerned, in addition to the four corners of the local-scale computational domain (numbered 1, 4, 7 and 10 in Fig. 8), we select two additional points for each lateral side of the domain and one point inside the domain. This latter is the WRF grid point (labelled S in Fig. 8) that is closer to the measurement station; more precisely, grid-point $S$ is 650 meters North-West of the measurement station and at a lower altitude above sea level: 650 vs. $761 \mathrm{~m}$.

As far as the time-averaging is concerned, two different discretizations are selected

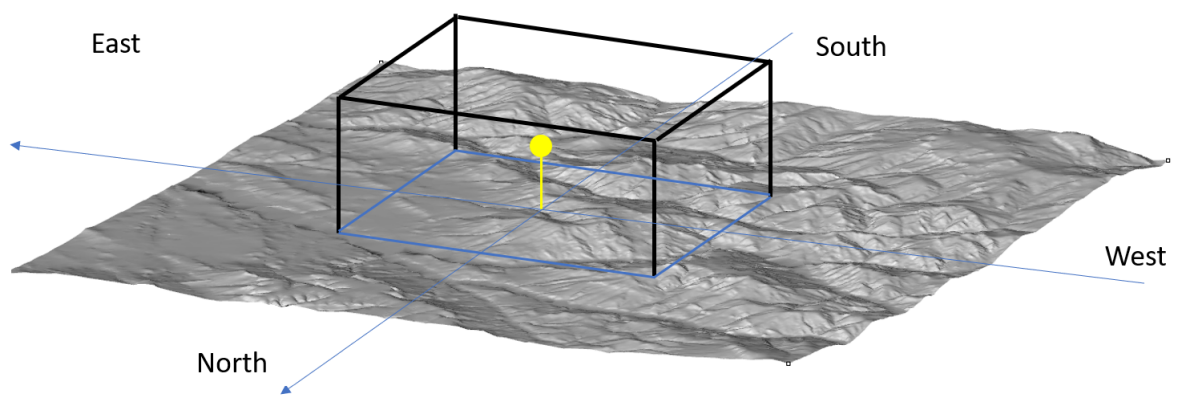

Figure 5: Local scale computational domain: measurement station location and edges of the box where mesh refinement is applied. 


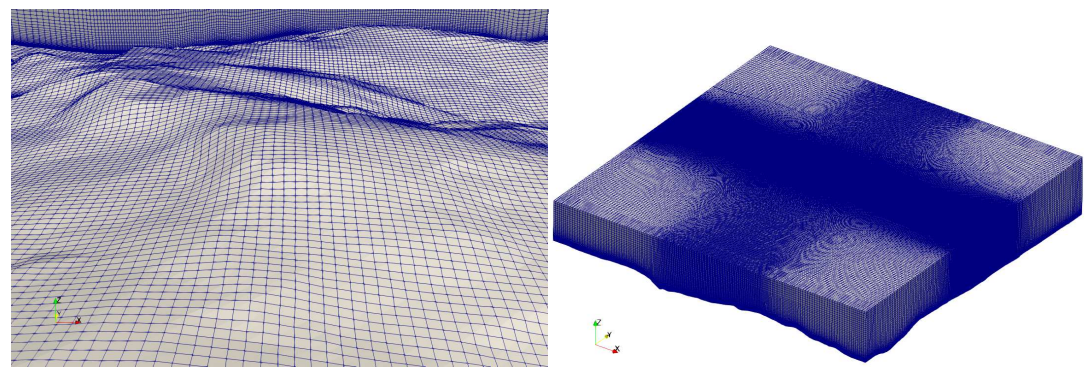

(a) Ground mesh

(b) Overall view showing mesh-refinement around the measurement station

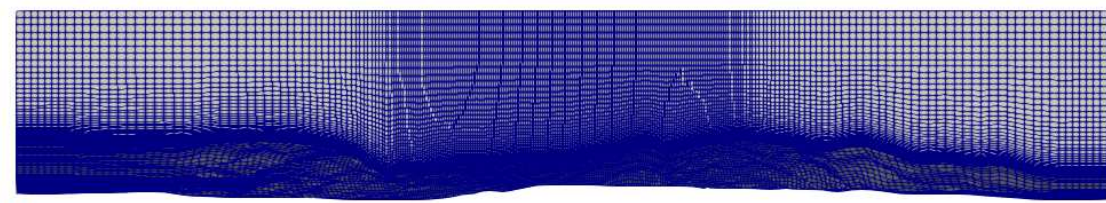

$\alpha$

$r x$

(c) CFD Mesh in the $y=0$ plane

Figure 6: Local scale computational grid (test case of October 19th, 2019).

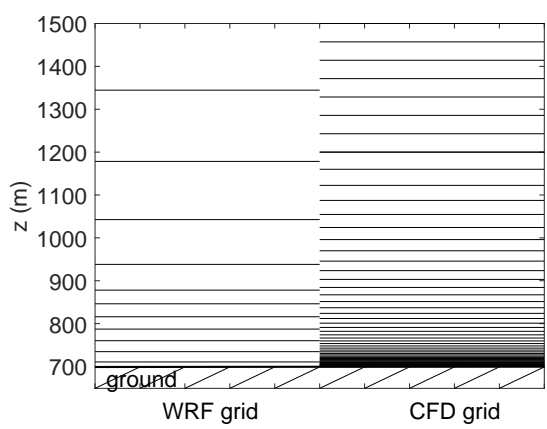

Figure 7: Vertical discretization used in the mesoscale and CFD grids (test case of October 19, 2019, SouthEast vertex) 
to verify the sensitivity of the prediction with respect to the time-averaging interval $M$ defined in Sect. 2.3. A coarse choice of 4 snapshot and a finer one of 8 snapshots are used to generate the time-averaged boundary conditions to be supplied to the local scale model. It is important to notice that a fundamental requirement of the proposed downscaling methodology is its computational affordability, so that it can be used alongside the NWP in operational forecasting. A too small period associated with the snapshots would result in too many CFD simulations and a too large demand in terms of computational requirements.

By reference to the nomenclature introduced in Sect. 2.3, we use an overlap $D=$ $50 \%$ and we split the time interval $T \approx 12$ hours for day " 1 ", and $T \approx 8$ hours for days "2" and "3") into either $N=4$ (coarse discretization) or $N=8$ (fine discretization) snapshots. In the former case we obtain four segments of length $M \approx 4.8$ hours for day "1", and $M \approx 3.2$ hours for the other days. In the latter case, we obtain eight segments of length $M=\approx 2.7$ hours for day "1" and $M \approx 1.8$ hours for days " 2 " and " 3 ".

For example, Fig. 9 shows the time evolution of the wind speed and direction, $10 \mathrm{~m}$

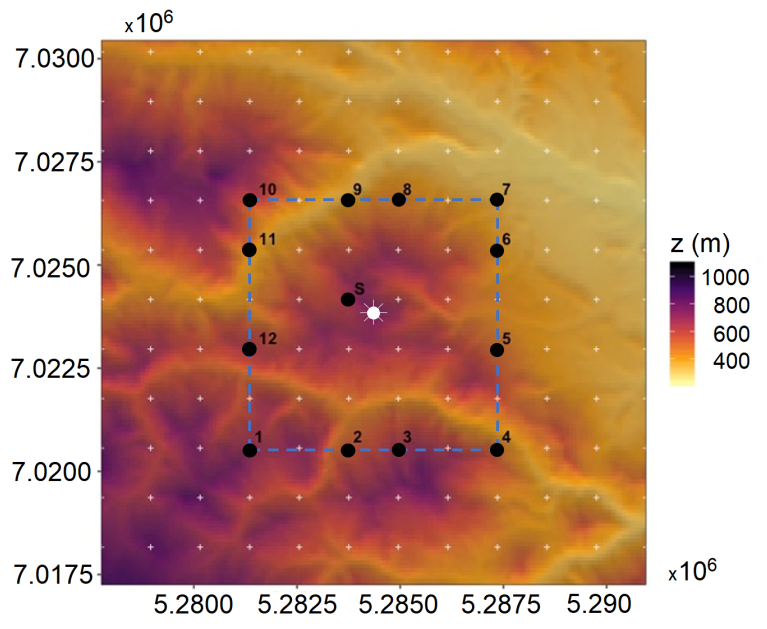

Figure 8: WRF grid-points plot on top of the DTM and numbering of the sampling points (black circles); the white marker at the center of the image corresponds to the measurement station. 
above the ground, obtained from the mesoscale simulation of day " 1 " in the sampling point $S$ (Fig. 87. Fig.9a and Fig.9b show the averaging method using 4 and 8 snapshots, respectively. Here, the markers represent the values of the averaged quantity, while the bars represent the extent of the averaging period considered for the snapshot. We use a reference angle $\theta$ to define the inflow direction that is $0^{\circ}$ when the wind vector is oriented from west to east, and its positive variation is counter-clockwise.

Time-averaging of the WRF output is automatically applied in all the sampling points shown in Fig. 8. The WRF data is saved on the first 15 pressure levels, which contain the diurnal development of the PBL above ground level.

The wind velocity profiles on the four lateral boundaries of the computational domain are obtained by interpolating the data from the sampling points to each lateral patch, as well as the top patch corresponding to the maximum PBLH. Fig. 10 shows the vertical profile of the time-averaged wind speed and direction, in the fifth snapshot of the fine time-discretization for each selected day. The values are taken at the center of the four boundary patches of the local scale domain. Close inspection of Fig. 10 reveals that, the wind has the usual shear profile within the surface layer, see Fig. 10 (left), but this suddenly changes in day " 1 ". The change in wind direction suggests that the flow above 1000 meters above sea level is stratified, featuring a gradual change of direction and magnitude, and this particular feature could be difficult to simulate by CFD using a standard RANS approach. For day "1", we investigate the effect of using two different RANS models (with and without the buoyancy terms and temperature equation) to downscale the prediction for the third snapshot of Fig. 9a, which ranges between around 13:00 and 17:00. This snapshot will be referred to as the "reference time period" and its specific analysis is reported in Section 4.2 .

Once the WRF wind velocity has been interpolated on the CFD domain boundaries, we found a relative mass flow rate unbalance of about $0.015 \%$ between inflow and outflow. We fixed it by applying one iteration of the procedure described in Sect. 2.4.2.

\subsubsection{Ground boundary conditions}

The wall function described in Sect. 2.4.3 is applied to the ground surface. To improve the fidelity of the simulation, we account for a heterogeneous distribution of 
roughness, based upon the actual land use in the area, which we retrieve from the GIS file of the land coverage $\AA^{4}$.

Table 2 reports the type of land coverage found in the selected area, associating each type to the corresponding CLC code [41] and equivalent sand roughness [42].

In order to convert the data from the GIS format to the OpenFOAM case directory we implemented a dedicated automatic procedure involving the Python Shapefile Library ${ }^{5}$ (PSL) and the TopoSet and CreatePatch pre-processing routines available in OpenFOAM. The details of the algorithm can be found in Appendix 2.

Fig. 11 shows the roughness distribution on the ground patch, following the application of the procedure described in Appendix 2.

\subsubsection{Solver and schemes}

The solution algorithm used for the computation is the Semi-Implicit Method for Pressure Linked Equations (SIMPLE) solver [50], as implemented in OpenFOAM v1806 [6]. As already mentioned in Sect. 2.2. we perform two sets of simulations. A first set is based upon the incompressible (constant density) RANS equations, cou-

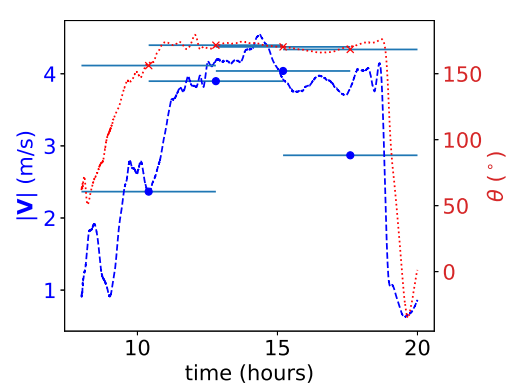

(a) 4 snapshots case

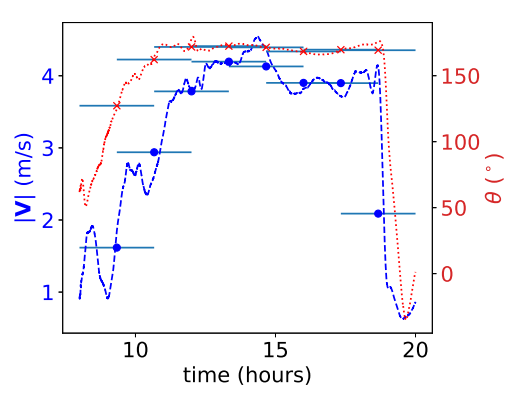

(b) 8 snapshots case

Figure 9: Time history, computed by WRF $10 \mathrm{~m}$ above the ground, of the wind speed (blue line) and direction (red line); averaged values (blue and red marks) and averaging time periods (horizontal lines).

\footnotetext{
${ }^{4}$ Source: http://www.sinanet.isprambiente.it/it/sia-ispra/download-mais/ corine-land-cover, Version: Corine Land Cover 2012 IV

$\sqrt[3]{\text { https://pythonhosted.org/PythonShapefileLibrary/ }}$
} 


\begin{tabular}{lcl}
\hline CLC12 & z0 & Description \\
\hline 112 & 0.5 & Residential areas \\
121 & 0.5 & Industrial areas \\
221 & 0.1 & Grape groves \\
223 & 0.1 & Olive groves \\
231 & 0.03 & Grasses \\
242 & 0.3 & Particle and complex cultivation systems \\
243 & 0.3 & Agrarian crops \\
324 & 0.05 & Shrubby vegetation \\
331 & 0.0003 & Sand dunes \\
512 & 0.0001 & Water surface \\
2111 & 0.05 & Intensive crops \\
2112 & 0.05 & Extensive crops \\
3112 & 0.75 & Caduceus leaves forests \\
3116 & 0.75 & Hygrophic trees forests \\
3121 & 0.75 & Pine forests \\
3231 & 0.75 & High thicket \\
\hline
\end{tabular}

Table 2: Land use categories in the selected area. 

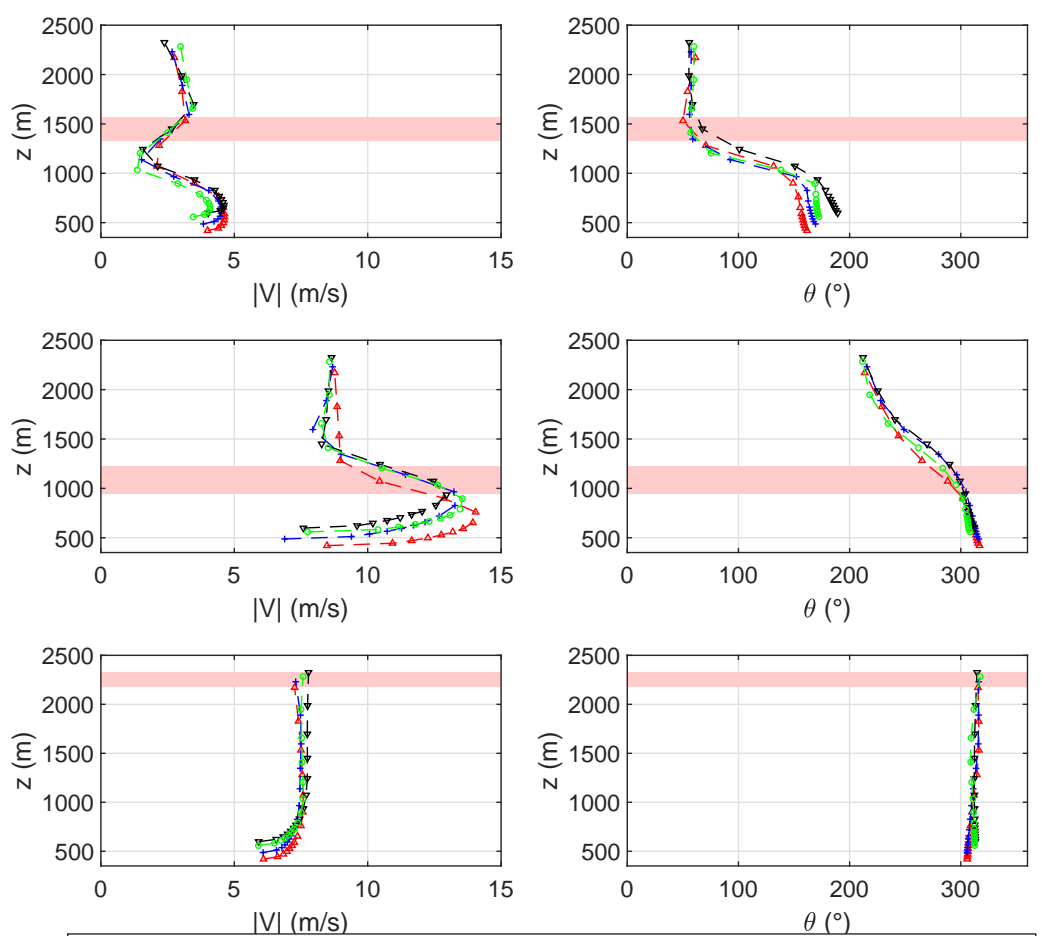

PBLH $-\rightarrow-$ - east side $-\rightarrow-\cdot$ north side $-\rightarrow-\cdot$ south side $-\rightarrow-$ west side

Figure 10: Averaged wind profile interpolated at the four lateral boundary patches. Left: Wind-speed magnitude vs. elevation above sea level. Right: Wind direction vs. elevation above sea level. Top row: day "1". Middle row: day "2". Bottom row: day "3"

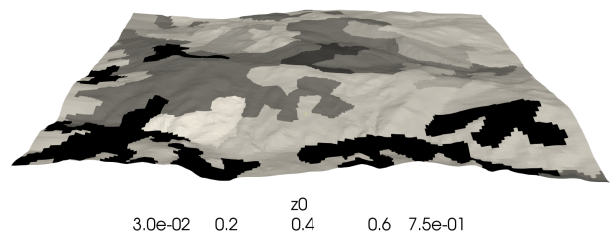

Figure 11: Roughness length distribution corresponding to different land use. 
pled with the standard k- $\varepsilon$ model. This model has been adopted to produce all the results presented in Section 4.1. A second model has also been tested and applied to the "reference time period" (defined in Sect. 3.4.2). This second approach allows to deal with incompressible stratified flows by including the effects of buoyancy, and also accounting for the energy balance equation in the form of a temperature transport equation [51; 50]. In this case, a boundary condition for the temperature should be also given in the set-up. Therefore, the temperature from the mesoscale grid points has been averaged and interpolated on the local scale boundary surfaces.

In the former case we use the simpleFoam solver while in the latter we use the buoyantBoussinesqSimpleFoam solver.

Concerning the spatial discretization, the linear-upwind scheme is used for the continuity equation, limited linear schemes are used for the convective terms of the turbulence transport equations, and the un-limited linear reconstruction is used for all the other terms in the governing equations (momentum and temperature).

Concerning the linear solvers, GAMG is used for the pressure-Poisson equation and PBiCG for all other equations.

A typical simpleFoam run for a snapshot requires about 4000 pseudo-time steps to converge to steady-state (residuals for all the equations ranging between $10^{-6}$ and $10^{-7}$ ); about 5000 pseudo-time steps are required when using buoyant Bous s inesqSimpleFoam solver. Iterative convergence is checked not only by looking at the residuals of the various equations, but also by making sure that the relative changes in the computed wind speed at the measurement point fall below a threshold pre-set by the user. 10 hours of wall clock time are required to run 10,000 simpleFoam pseudo-time steps on a quad-core machine, around $20 \%$ more is needed on the same hardware for the buoyantBoussinesqSimpleFoam solver. 


\section{Results}

\subsection{Wind prediction downscaling using different averaging periods on all three days}

The prediction of the wind in the three sample days has been downscaled using the two sets of averaged snapshots defined in Section 3.4.2. Fig. 12 reports the local-scale (CFD) prediction of the wind velocity and direction at the measurement point, compared with the time history of the measurements. These plots show the accuracy of the prediction, but also its sensitivity with respect to the time-averaging period, i.e. the number of snapshots. As expected, a finer discretization results in a prediction closer to the measurement. The boundary condition, obtained by averaging the mesoscale solution, depart from the instantaneous condition when the averaging period is relatively too large. We must say "relatively" indeed, because when the time-variation of the wind is small (day "2" and "3") this difference reduces. In day "1", the direction and magnitude of the wind shows stronger variations in time and the sensitivity to the averaging period is more evident. This observation suggests that an adaptive method to compute the averaging period of each snapshot might be a better choice, because a non-uniform discretization of the period could better follow the time variation of the wind field, thus improving accuracy while retaining a limited number of local scale simulations.

We select the fifth snapshot for each simulated day to investigate the flow features predicted by the local scale model. Fig. 13 shows the turbulent kinetic energy and wind velocity on a horizontal slice of the domain, taken at 800 meters above sea level.

For day "2" and "3" we notice a similar situation, with a strong wind coming from North-East. The turbulence develops in correspondence of the peaks of the mountains and it is transported downstream by the flow.

The case of day "1" appears different with respect to the other two. We notice a large production of turbulence close to the North and West boundaries. The velocity field is characterized by a much weaker wind, which is slowed down after crossing the mountains at the center of the field. Taking a look to the wind profile of day "1" obtained from the mesoscale simulation (Fig. 10, we see that inside the PBL there is a mixing between two main currents. The first, close to the ground and blowing 

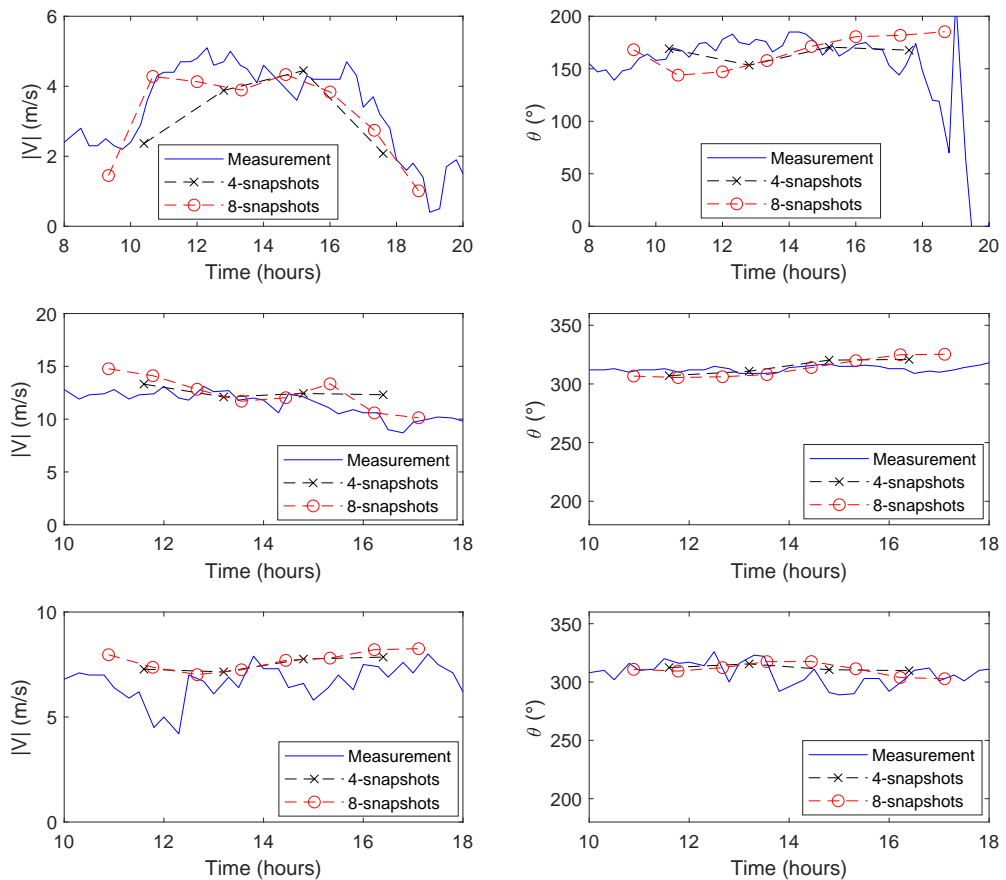

Figure 12: Prediction of the wind velocity and direction at the measurement point for two sets of averaging snapshots (cross markers: 4 snapshots set, circle marker: 8 snapshots set). The continuous line represents the measurements. Top: day "1". Middle: day "2". Bottom: day "3"

toward West, the other, blowing toward North-East at higher altitudes. We argue that, in this case, the local scale solution requires a higher turbulent kinetic energy (and viscosity), to guarantee the fulfilment of this complex boundary condition prescribed to the velocity field at all altitudes.

\subsection{Wind prediction downscaling using different RANS models on day "I"}

As explained in Sect 2.2 two different sets of simulations have been performed on day "1", and will be described hereafter, which differ by the fact that buoyancy effects are, or are not, accounted for.

In the former case, the addition of the temperature equation and the introduction of the gravity terms in the governing PDEs is expected to improve the description of flow 

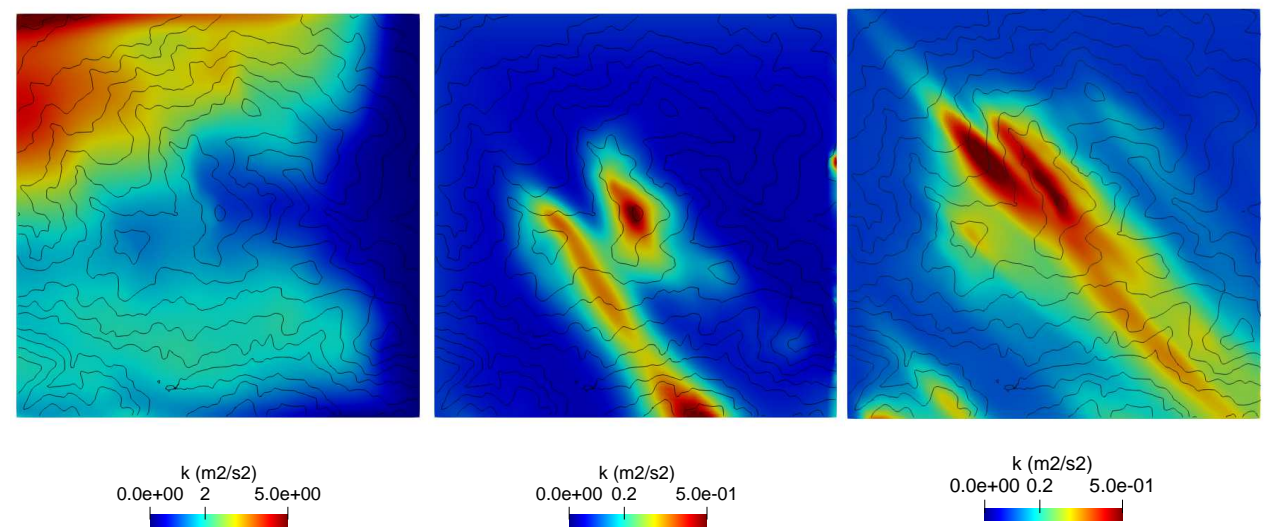

1

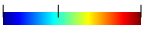

1
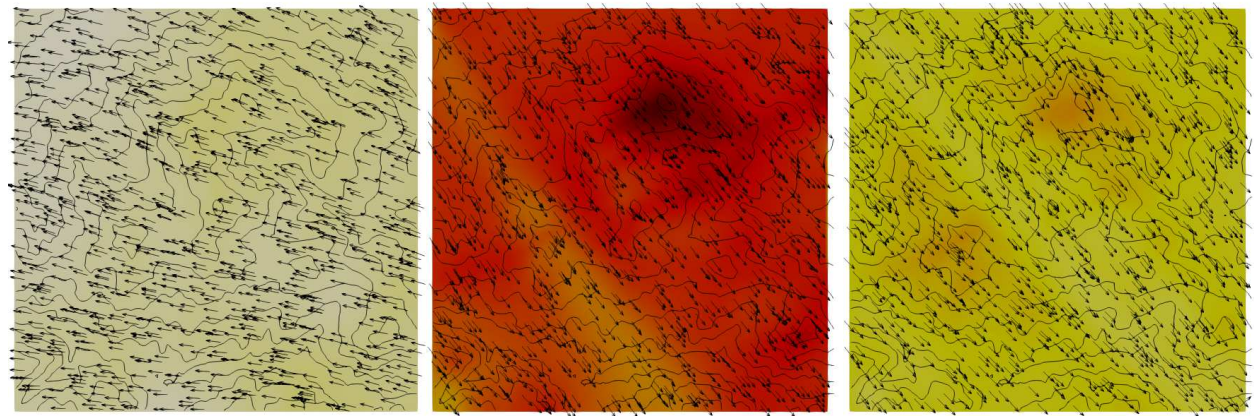

$2.0 \mathrm{e}+00 \stackrel{|\mathrm{V}|(\mathrm{m} / \mathrm{s})}{10} 1.6 \mathrm{e}+01$
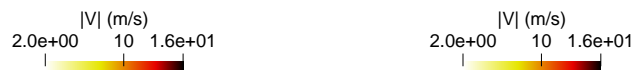

Figure 13: Turbulent kinetic energy (top row) and wind velocity (bottom row) at $800 \mathrm{~m}$ above sea level. The sample snapshot is relative to the 16 UTC. Left: day "1". Center: day "2". Right: day "3". The black lines represent the ground elevation contour lines every 50 meters.

stratification.

We verify the differences in the results obtained for the third snapshot of day "1" (reference time period), defined in Sect. 3.4.2.

Fig. 14 shows a view of the wind velocity vectors over two mutually perpendicular planes which slice the domain, passing through the measurement station, i.e. the point flagged in Fig. 5 We notice that both modelling options are capable of preserving throughout the inner domain the wind velocity profile prescribed by the mesoscale 
boundary conditions. However, when the buoyancy term are also accounted for in the equations it is possible to predict a more pronounced variation of the wind direction close to the ground, especially where the orographic profile is characterized by gorges and valleys: see the green rectangles in Fig. 14

It is worth underlining that a wind velocity profile featuring such a stratification and variation of the direction along the height, which is not rare in atmospheric flows, may be very difficult to simulate using 3D steady RANS modelling. Therefore, the present computation also allows us to establish to which extent this standard CFD technique is capable of coping with this particular flow pattern.

Fig. 15 shows the turbulent kinetic energy $k$ within the first cell above the ground, in the area surrounding the measurement station. Here, the differences between the two different modelling options are even more pronounced. The simulation that accounts for buoyancy reveals, in general, a higher production of turbulent kinetic energy over the peaks of the mountains (Fig. 15c), and in the area located North-West of the measurement station.

It is also possible to observe the influence of the local ground roughness on the turbulent kinetic energy field. Referring to Fig. 15a, which shows the equivalent roughness distribution, we expect a higher production of $k$ due to the land coverage in the South-East areas close to the measurement station, where some building and structures are present, while less turbulence is expected in the North-West and far South areas.

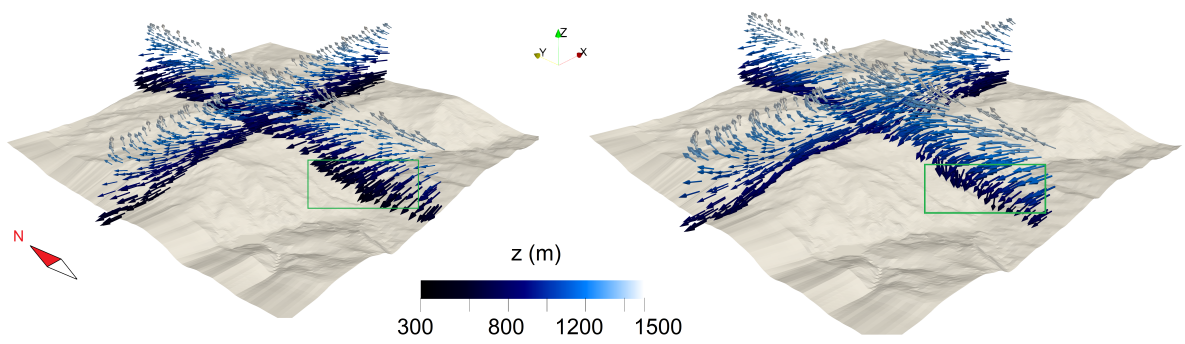

Figure 14: Wind velocity vector field on two mutually perpendicular planes slicing the computational domain. The rectangle highlights the region where larger differences between the two modelling options can be seen. Left: Not accounting for buoyancy. Right: Accounting for buoyancy. 

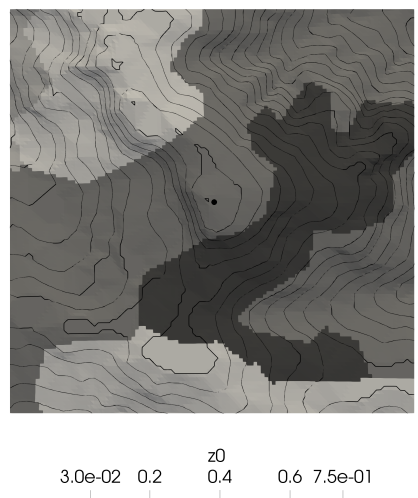

(a) Equivalent ground roughness.

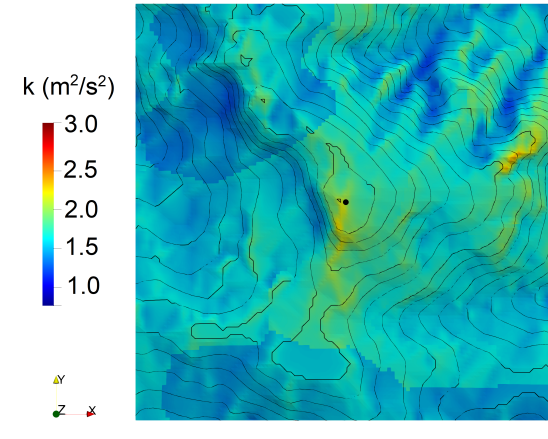

(b) Not accounting for buoyancy.

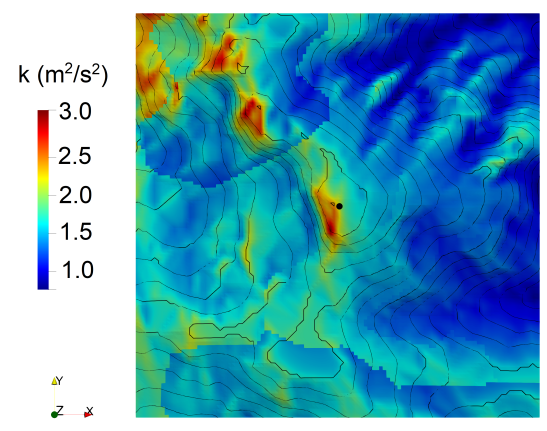

(c) Accounting for buoyancy.

Figure 15: Turbulent kinetic energy and equivalent roughness at the ground in a neighbourhood $(1.5 \times 1.5$ $\mathrm{km}$ ) of the measurement station. The black lines represent the ground elevation contour lines (every 10 meters).

We can notice this effect as a slight discontinuity between the regions with different roughness, visible in the areas where $k$ has a small value (Fig. $15 \mathrm{~b}$ and Fig. $15 \mathrm{c}$ ).

Fig. 16 shows the wind speed and direction profiles at the measurement station and at two points located $100 \mathrm{~m}$ upwind and downwind along the East-West direction. The profiles are plotted throughout the entire height of the computational domain. The comparison among the profiles computed at the three different locations shows that the local orography is felt within the first 200 meters of the profile, whereas almost 
identical profiles can be seen further away from the ground. Minor differences between the profiles can also be seen depending on whether the buoyancy terms are accounted for, or not.

The verification of the calculation is made by comparing the RANS result against the wind speed and direction measured at the anemometer station and averaged over the same time interval used to generate the snapshot. Table 3 compares the observed data against the RANS simulations using the two different modelling options. The agreement between the simulated results and the measured wind speed is fairly good. The relative error with respect to the wind velocity is $1.59 \%$ for the simulation not accounting for buoyancy, and $0.68 \%$ for the simulation accounting for buoyancy. The average flow direction shows a larger discrepancy between measurements and simulations. The relative error in this case is $2.53 \%$ for the first simulation and $2.86 \%$ for the second one.

Grid point $S$ of Fig. 8 (the WRF grid point closer to the measurement station) has been used to examine the down-scaling in closer detail by comparing the wind velocity profiles (magnitude and direction) obtained from the mesoscale and local scale simulations. The three sets of simulations are compared in Fig. 17

Differences can be seen both at low and high altitudes. Close to the ground, the wind predicted by the two CFD simulations is characterized by a lower speed and it is more North-bound than that predicted by WRF. The effect of the ground on the profile appears to be stronger in the RANS computation that does not account for buoyancy. At higher altitude we observe that in both CFD simulations the wind profile prescribed at the boundary is not perfectly preserved, while we would expect that the mesoscale and local scale prediction would give a very similar result in this region. In the absence of measured data at these altitudes, we can only infer that this behaviour is related to an overestimated mixing effect of the wind layers in the 3D field computed by RANS.

In the end, if we compare the value of $k$ predicted by the mesoscale model at the sampling point $S\left(k_{W R F}^{(S)}=0.29 \mathrm{~m}^{2} / \mathrm{s}^{2}\right)$, with the one obtained from the RANS simulations $\left(k_{R A N S_{1}}^{(S)}=2.41 \mathrm{~m}^{2} / \mathrm{s}^{2}, k_{R A N S_{2}}^{(S)}=4.49 \mathrm{~m}^{2} / \mathrm{s}^{2}\right)$ we find that the local scale RANS predicts a sensibly higher value of turbulent kinetic energy. A sudden increase of the turbulent kinetic energy downstream of the inflow boundary was observed also in [10], 
where this effect was attributed to the inconsistency between the mesoscale and local scale governing equations [52].

The results presented so far, along with the validation test-case reported in Appendix 1, suggest that the reliability of the CFD RANS predictions, especially at higher altitude, deteriorates as the inflow profile departs from the theoretical one, given by Eq. (1).

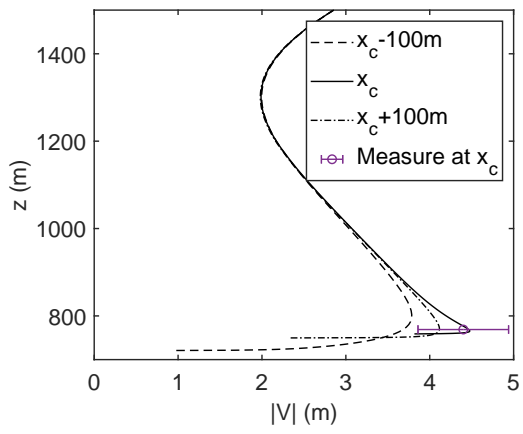

(a) Not accounting for buoyancy.

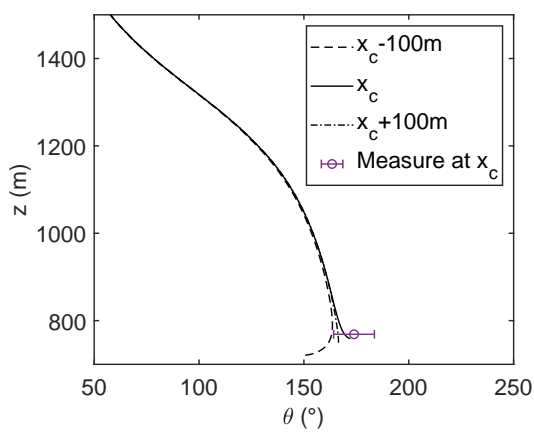

(c) Not accounting for buoyancy.

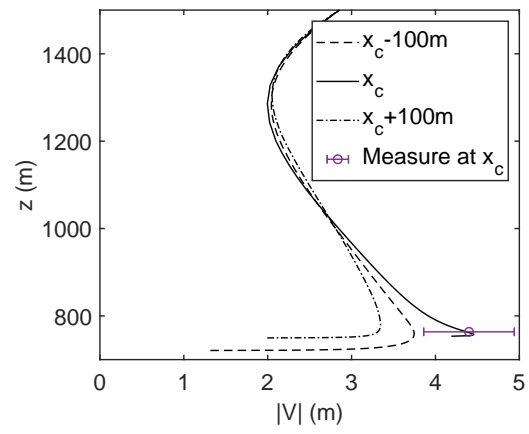

(b) Accounting for buoyancy.

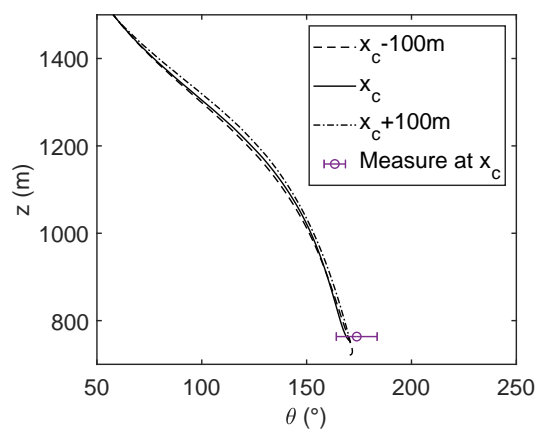

(d) Accounting for buoyancy.

Figure 16: Wind velocity and direction profiles at and close to the measurement station. 


\begin{tabular}{lcccc}
\hline Case & Wind speed $(\mathrm{m} / \mathrm{s})$ & Direction $\left(^{\circ}\right)$ & $\sigma_{V}(\mathrm{~m} / \mathrm{s})$ & $\sigma_{\theta}\left({ }^{\circ}\right)$ \\
\hline Measurement & 4.40 & 173.9 & 0.36 & 6.49 \\
RANS w/o buoyancy & 4.47 & 169.5 & - & - \\
RANS with buoyancy & 4.37 & 168.9 & - & - \\
\hline
\end{tabular}

Table 3: Validation study: RANS calculations against measured data at the measurement station. Standard deviation of the wind velocity $\sigma_{V}$ and direction $\sigma_{\theta}$ is provided for the averaged data.

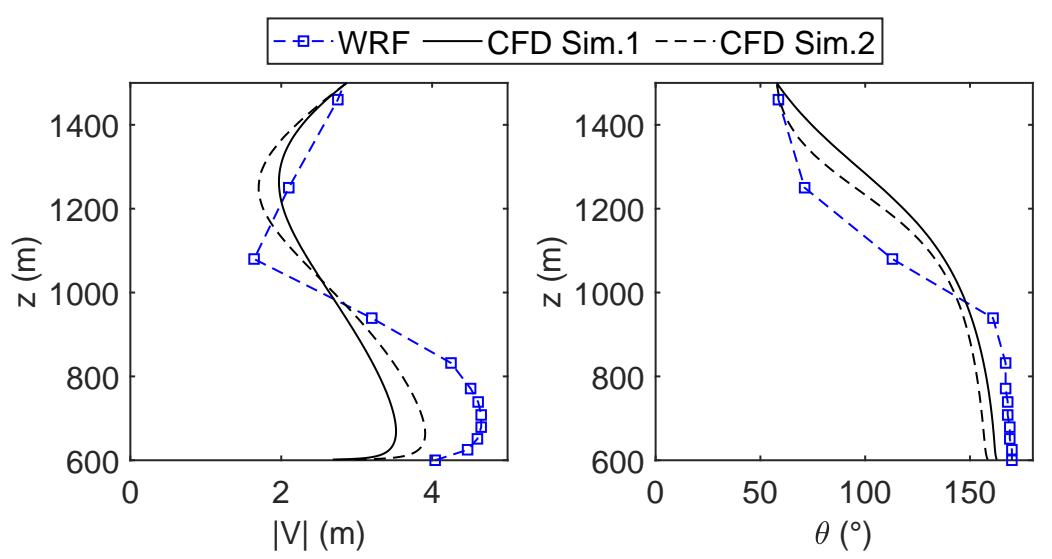

Figure 17: Comparison between the wind velocity profiles (magnitude and direction) computed using the meso-scale and the two local-scale simulations (1: w/o buoyancy; 2: accounting for buoyancy) at grid point $S$. 


\section{Concluding remarks}

In this paper we have presented a multiscale methodology that allows to couple the mesoscale prediction of the wind with a CFD model for turbulent flow simulations. The combined use of these two different methodologies allows to increase the spatial resolution of the wind forecast, averaged over a time interval of the order of a few hours.

To make the methodology easily replicable, we rely on two different opensource platforms for simulating the wind at the mesoscale (WRF-ARW) and local-scale (OpenFOAM) level. Moreover, to preserve the usability of the procedure through future software updates and releases, the approaches developed to interface the two simulation tools rely on pre- and post-processing data structures and routines already available for these two software suites. In particular, we propose methods for: i) time-averaging the NWP simulation data to be used as boundary conditions for the local-scale simulation; ii) coupling the two solvers at the boundary faces of the CFD domain; iii) accounting for variable land use in terms of local roughness, starting from the GIS vector representation of the land usage.

The method has been tested on a site located in southern Italy and the results of the simulations have been verified against the anemometric data available three case studies between 2019 and 2020. Each day is representative of a windy day in a different season. The forecast have been averaged using two different time resolutions, verifying how the selection of the optimal averaging period is related to the time variability of the wind in the day. The discretizations adopted in the study have been found suitable for most of the analyses, except for the first and last parts of day "1", which exhibited strong wind variations in few hours.

The same day is also characterized by a complex shape of the planetary boundary layer. This situation has been taken as reference to test two different physicalmodelling options for the local-scale simulation. These include: the standard k- $\varepsilon$ RANS model for incompressible flows and a variation which includes the effects of buoyancy by accounting temperature variations. The comparison between measurements and the local-scale (CFD) simulations suggests that accounting for buoyancy 
effects slightly improves the simulation fidelity, but the experimental data are only available at $10 \mathrm{~m}$ above the ground, so that further validation against experimental data is certainly required.

We conclude that the downscaling approach increases the detail of the wind prediction supplied by the mesoscale model in the target area, and in particular close to the ground, because the local-scale simulation relies of a finer spatial resolution and a significantly higher detail in the model of the terrain geometry. However, far above the ground RANS modelling seems to run into troubles when trying to cope with the complex PBL shape supplemented as boundary condition by the mesoscale simulation.

\section{Acknowledgment}

The authors acknowledge the European Union and the Italian Ministry of Research for financial support under the Programma Operativo Nazionale Ricerca e Innovazione 2014-2020, CUP - 34I19000050006, Activity - AIM 1859451 / 3. 


\section{Appendix 1: Validation using the Askervein hill case}

We tested the accuracy of the local-scale methodology by reference to a wellestablished benchmark case: the so-called Askervein hill case study, which has been used by many researchers (e.g. [10; 16]) as a validation/verification case for PBL numerical simulations.

Table 4 gives the location of the site and of the measurement line. Detailed measurements obtained by Taylor et al. in the framework of the Askervein hill project can be found in [53: 54].

We used the same numerical setting, including boundary conditions and terrain model, also used in [10]; further details are given in Tab. 5. Observe that the inlet inflow profile has been analytically prescribed using Eq. (1) and has not been obtained from a meso scale simulation. Table 6 provides information concerning the two different, un-nested, computational grids that have been used for this validation exercise.

Figure 18 shows the comparison between the measured data and the RANS solutions (without accounting for buoyancy effects) computed using the two different grids. The agreement is fair and in line with similar studies reported in the literature. Apart from the simulated turbulent kinetic energy downhill, which departs from the measurements, the wind velocity components computed on the two different grids follow the experimental trends and fall within the error uncertainty of the experiments.

\section{Appendix 2: Land coverage application algorithm for OpenFoam}

In this Appendix we give details concerning the algorithm that has been coded to account for a variable land use, as described in Sect.2.4.3.

We start by reading the GIS file containing the land coverage information. For each zone the algorithm proceeds as follows: i) extract the polyline of the zone boundary on the ground; ii) project the points on the coordinate system of the CFD domain, and on a plane with $Z<Z_{\min }$ (where $Z_{\min }$ is the minimum ground elevation); iii) create a constrained Delaunay triangulation of the polyline internal area; iv) extrude the area along the $Z$ direction until a plane $Z>Z_{\max }$ (where $Z_{\max }$ is the maximum ground elevation in the area); v) export the bounding surface as an STL file; vi) use toposet to create 


\begin{tabular}{lr}
\hline \multicolumn{2}{c}{ Site description } \\
\hline Country & Scotland \\
Latitude & $57^{\circ} 11^{\prime} \mathrm{N}$ \\
Longitude & $7^{\circ} 22^{\prime} \mathrm{W}$ \\
Altitude & $126 \mathrm{~m}$ \\
\hline \multicolumn{2}{c}{ Measurement line A } \\
\hline Height from ground & $10 \mathrm{~m}$ \\
Orientation & $43^{\circ}$ (from WE line) \\
Origin & Hill highest point \\
\hline
\end{tabular}

Table 4: Askervein hill project data.

\begin{tabular}{lr}
\hline \multicolumn{2}{c}{ Reference wind inflow } \\
\hline Measurement period & $03 / 10 / 1983$, h $14: 00-17: 00$ \\
Uref & $9.11 \mathrm{~m} / \mathrm{s}$, Regular \\
Zref & $10 \mathrm{~m}$ \\
Angle & $210^{\circ}$ \\
\hline & CFD Domain and BC \\
\hline Domain orientation & Inflow aligned \\
Sizes & $6 \times 6 \times 1$ km ${ }^{3}$ \\
Inflow Patch BC & PBL model \\
Sides and Top Patches BC & Not-permeability (slip) \\
Outflow Patch BC & Neumann zero-gradient \\
Terrain & Rough PBL wall function, $\mathrm{z}_{0}=0.035$ \\
\hline
\end{tabular}

Table 5: Domain and boundary conditions.

an OpenFoam face zone by intersecting the ground boundary patch with the surface of the STL file; vii) use createPatch to generate a named wall patch; viii) update the boundary condition files with the new patch, associating to it the roughness value $z_{0}$, given by mapping the land use code saved in the GIS file with the corresponding 


\begin{tabular}{lcc}
\hline & Mesh 1 & Mesh 2 \\
\hline Type & Snappy hex mesh & Structured mesh \\
N cells & $9.5 \mathrm{MLN}$ & $3.2 \mathrm{MLN}$ \\
Min/Max DX, DY & $10 \mathrm{~m} / 85 \mathrm{~m}$ & $20 \mathrm{~m} / 80 \mathrm{~m}$ \\
Min/Max DZ, DY & $0.6 \mathrm{~m} / 72 \mathrm{~m}$ & $1 \mathrm{~m} / 50 \mathrm{~m}$ \\
Cells in the first $100 \mathrm{~m}$ & 25 & 25 \\
\hline
\end{tabular}

Table 6: Askervein hill simulation. Mesh 1 and Mesh 2 details.

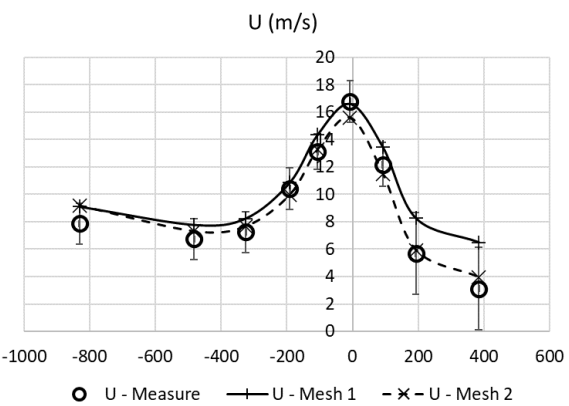

(a) Wind-velocity component in the $x$ direction.

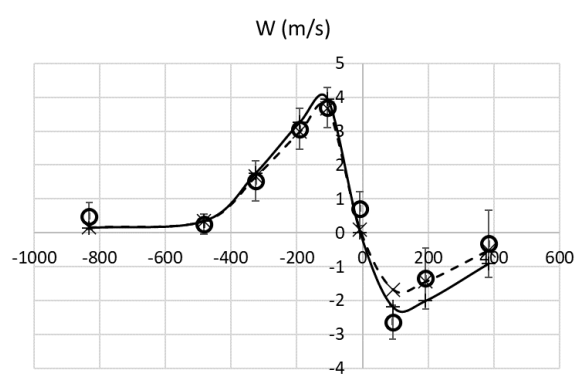

O W-Measure —-w - Mesh $1-*-w-$ Mesh 2

(b) Wind-velocity component in the vertical direction.

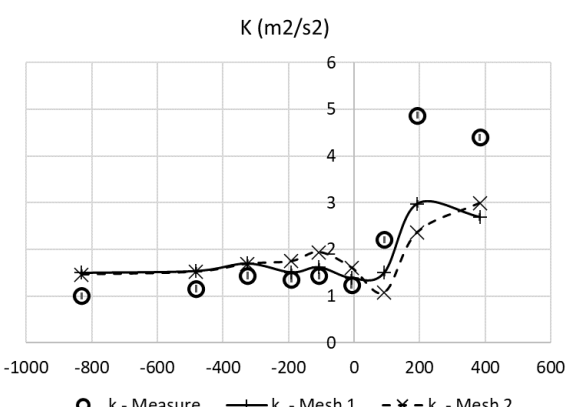

○ k-Measure $\quad-k-$ Mesh $1 \quad-*-k-$ Mesh 2

(c) Turbulent kinetic energy of the wind.

Figure 18: Askervein hill simulation results.

equivalent sand roughness [42].

The aforementioned procedure is automatically iterated for all the zones describing the land use in the area. 


\section{References}

[1] W.C. Skamarock, J.B. Klemp, J. Dudhia, D.O. Gill, D.M. Barker, W. Wang, and J.G. Powers, "A description of the advanced research wrf version 3. ncar technical note-475+ str", (2008).

[2] W.C. Skamarock, J.B. Klemp, J. Dudhia, D.O. Gill, Z. Liu, J. Berner, W. Wang, J.G. Powers, M.G. Duda, D.M. Barker, et al., "A description of the advanced research wrf model version 4", Natl. Ctr. Atmos. Res., Boulder, CO, 7 (2008).

[3] A. Castorrini, A. Corsini, F. Rispoli, P. Venturini, K. Takizawa, and T.E. Tezduyar, "Computational analysis of performance deterioration of a wind turbine blade strip subjected to environmental erosion”, Computational Mechanics, published online, DOI: 10.1007/s00466-019-01697-0, March 2019, doi: 10.1007/s00466019-01697-0.

[4] A. Corsini, A. Castorrini, E. Morei, F. Rispoli, F. Sciulli, and P. Venturini, "Modeling of rain drop erosion in a multi-MW wind turbine", in ASME Turbo Expo, Montreal, Canada, (2015).

[5] D. Mehta, A. Van Zuijlen, B. Koren, J. Holierhoek, and H. Bijl, "Large eddy simulation of wind farm aerodynamics: A review", Journal of Wind Engineering and Industrial Aerodynamics, 133 (2014) 1-17.

[6] H.G. Weller, G. Tabor, H. Jasak, and C. Fureby, “A tensorial approach to computational continuum mechanics using object-oriented techniques”, Computers in physics, 12 (1998) 620-631.

[7] Y. Miao, S. Liu, B. Chen, B. Zhang, S. Wang, and S. Li, "Simulating urban flow and dispersion in Beijing by coupling a CFD model with the WRF model", Advances in Atmospheric Sciences, 30 (2013) 1663-1678, doi: 10.1007/s00376013-2234-9.

[8] Y. Miao, S. Liu, H. Zheng, Y. Zheng, B. Chen, and S. Wang, "A multi-scale urban atmospheric dispersion model for emergency management", Advances in Atmospheric Sciences, 31 (2014) 1353-1365, doi: 10.1007/s00376-014-3254-9. 
[9] S. Montelpare, V. D’Alessandro, C. Lops, E. Costanzo, and R. Ricci, “A mesoscale-microscale approach for the energy analysis of buildings", in Journal of Physics: Conference Series, volume 1224. IOP Publishing, (2019) 012022.

[10] O. Temel, L. Bricteux, and J. van Beeck, "Coupled wrf-openfoam study of wind flow over complex terrain", Journal of Wind Engineering and Industrial Aerodynamics, 174 (2018) 152-169.

[11] M. Tewari, H. Kusaka, F. Chen, W.J. Coirier, S. Kim, A.A. Wyszogrodzki, and T.T. Warner, "Impact of coupling a microscale computational fluid dynamics model with a mesoscale model on urban scale contaminant transport and dispersion”, Atmospheric Research, 96 (2010) 656-664.

[12] P. Durán, C. Mei $\beta$ ner, and P. Casso, "A new meso-microscale coupled modelling framework for wind resource assessment: A validation study", Renewable Energy, 160 (2020) 538-554.

[13] J.-J. Baik, S.-B. Park, and J.-J. Kim, "Urban flow and dispersion simulation using a cfd model coupled to a mesoscale model", Journal of Applied Meteorology and Climatology, 48 (2009) 1667-1681.

[14] C.V. Rodrigues and J. Palma, "Estimation of turbulence intensity and shear factor for diurnal and nocturnal periods with an urans flow solver coupled with wrf”, in Journal of Physics: Conference Series, volume 524. IOP Publishing, (2014) 012115.

[15] A.A. Wyszogrodzki, S. Miao, and F. Chen, "Evaluation of the coupling between mesoscale-wrf and les-eulag models for simulating fine-scale urban dispersion”, Atmospheric research, 118 (2012) 324-345.

[16] J. Sanz Rodrigo and P. Gankarski. "Windbench: Benchmarking of flow models for wind applications", 2014.

[17] P. Richards and R. Hoxey, "Appropriate boundary conditions for computational wind engineering models using the $\mathrm{k}-\varepsilon$ turbulence model", in Computational Wind Engineering 1, 145-153, Elsevier, 1993. 
[18] A. Parente, C. Gorlé, J. Van Beeck, and C. Benocci, "Improved k- $\varepsilon$ model and wall function formulation for the rans simulation of abl flows", Journal of wind engineering and industrial aerodynamics, 99 (2011) 267-278.

[19] B. Blocken, A. van der Hout, J. Dekker, and O. Weiler, "Cfd simulation of wind flow over natural complex terrain: case study with validation by field measurements for ria de ferrol, galicia, spain”, Journal of Wind Engineering and Industrial Aerodynamics, 147 (2015) 43-57.

[20] M. Balogh, A. Parente, and C. Benocci, "Rans simulation of abl flow over complex terrains applying an enhanced $\mathrm{k}-\varepsilon$ model and wall function formulation: Implementation and comparison for fluent and openfoam", Journal of Wind Engineering and Industrial Aerodynamics, 104 (2012) 360-368.

[21] A. Murali and R. Rajagopalan, "Numerical simulation of multiple interacting wind turbines on a complex terrain", Journal of Wind Engineering and Industrial Aerodynamics, 162 (2017) 57-72.

[22] F. Castellani, D. Astolfi, M. Burlando, and L. Terzi, "Numerical modelling for wind farm operational assessment in complex terrain", Journal of Wind Engineering and Industrial Aerodynamics, 147 (2015) 320-329.

[23] H. Gopalan, C. Gundling, K. Brown, B. Roget, J. Sitaraman, J.D. Mirocha, and W.O. Miller, "A coupled mesoscale-microscale framework for wind resource estimation and farm aerodynamics", Journal of Wind Engineering and Industrial Aerodynamics, 132 (2014) 13 - 26, doi: https://doi.org/10.1016/j.jweia.2014.06.001

[24] R.B. Bird, W.E. Stewart, and E.N. Lightfoot, "Transport phenomena john wiley \& sons", New York, 413 (1960).

[25] W. Wang, C. Bruyère, M. Duda, J. Dudhia, D. Gill, M. Kavulich, K. Keene, M. Chen, H. Lin, J. Michalakes, et al., "Wrf-arw version 3 modeling system user's guide", National Centre for Atmospheric Research, (2009). 
[26] V. Lara-Fanego, J. Ruiz-Arias, D. Pozo-Vázquez, F. Santos-Alamillos, and J. Tovar-Pescador, "Evaluation of the wrf model solar irradiance forecasts in andalusia (southern spain)", Solar Energy, 86 (2012) 2200-2217.

[27] R. Perez and T. Hoff, "Solar anywhere forecasting", Solar Energy Forecasting and Resource Assessment. Elsevier, (2013).

[28] E. Lorenz, J. Remund, S.C. Müller, W. Traunmüller, G. Steinmaurer, D. Pozo, J. Ruiz-Arias, V.L. Fanego, L. Ramirez, M.G. Romeo, et al., "Benchmarking of different approaches to forecast solar irradiance", in 24th European photovoltaic solar energy conference. Hamburg, Germany, (2009) 21-25.

[29] T.L. Feudo, E. Avolio, D. Gullì, S. Federico, C.R. Calidonna, and A.M. Sempreviva, "Comparison of hourly solar radiation from a ground-based station, remote sensing and weather forecast models at a coastal site of south italy (lamezia terme)", Energy Procedia, 76 (2015) 148-155.

[30] S. Gentile, F. Di Paola, D. Cimini, D. Gallucci, E. Geraldi, S. Larosa, S.T. Nilo, E. Ricciardelli, E. Ripepi, M. Viggiano, et al., "3d-var data assimilation of seviri radiances for the prediction of solar irradiance in italy using wrf solar mesoscale model-preliminary results", Remote Sensing, 12 (2020) 920.

[31] D. Carvalho, A. Rocha, M. Gómez-Gesteira, and C.S. Santos, "Wrf wind simulation and wind energy production estimates forced by different reanalyses: Comparison with observed data for portugal", Applied Energy, 117 (2014) 116-126.

[32] S. Shimada and T. Ohsawa, "Accuracy and characteristics of offshore wind speeds simulated by wrf", Sola, 7 (2011) 21-24.

[33] D. Carvalho, A. Rocha, M. Gómez-Gesteira, and C. Santos, "A sensitivity study of the wrf model in wind simulation for an area of high wind energy", Environmental Modelling \& Software, 33 (2012) 23-34.

[34] Y. Liu, T. Warner, Y. Liu, C. Vincent, W. Wu, B. Mahoney, S. Swerdlin, K. Parks, and J. Boehnert, "Simultaneous nested modeling from the synoptic scale to the les 
scale for wind energy applications", Journal of Wind Engineering and Industrial Aerodynamics, 99 (2011) 308-319.

[35] J.K. Lundquist, J. Mirocha, and B. Kosovic, "Nesting large-eddy simulations within mesoscale simulations in wrf for wind energy applications", in Proceedings of the Fifth International Symposium on Computational Wind Engineering, Chapel Hill, NC, May, (2010) 23-27.

[36] D. Hargreaves and N.G. Wright, "On the use of the $\mathrm{k}-\varepsilon$ model in commercial cfd software to model the neutral atmospheric boundary layer", Journal of wind engineering and industrial aerodynamics, 95 (2007) 355-369.

[37] S.B. Pope. "Turbulent flows", 2001.

[38] C. Alinot and C. Masson, "Aerodynamics of wind turbines in thermally stratified turbulent atmospheric boundary layer", in Proceedings of the 10th Annual Conference of the CFDSC, (2002) 553-559.

[39] L. Bernardet, J. Wolff, L. Nance, A. Loughe, B. Weatherhead, E. Gilleland, and B. Brown, "Comparison between wrf-arw and wrf-nmm objective forecast verification scores", in 23rd Conference on Weather Analysis and Forecasting/19th Conference on Numerical Weather Prediction. Omaha, NE. Accessed June, volume 13, (2009) 2016.

[40] P. Welch, "The use of fast fourier transform for the estimation of power spectra: a method based on time averaging over short, modified periodograms", IEEE Transactions on audio and electroacoustics, 15 (1967) 70-73.

[41] M. Bossard, J. Feranec, J. Otahel, et al., "Corine land cover technical guide: Addendum 2000", (2000).

[42] J. Silva, C. Ribeiro, and R. Guedes, "Roughness length classification of corine land cover classes", in Proceedings of the European Wind Energy Conference, Milan, Italy, volume 710. Citeseer, (2007) 110. 
[43] G. Thompson and T. Eidhammer, "A study of aerosol impacts on clouds and precipitation development in a large winter cyclone", Journal of the atmospheric sciences, 71 (2014) 3636-3658.

[44] E.J. Mlawer, S.J. Taubman, P.D. Brown, M.J. Iacono, and S.A. Clough, "Radiative transfer for inhomogeneous atmospheres: Rrtm, a validated correlatedk model for the longwave", Journal of Geophysical Research: Atmospheres, 102 (1997) 16663-16682.

[45] J. Dudhia, "Numerical study of convection observed during the winter monsoon experiment using a mesoscale two-dimensional model", Journal of the atmospheric sciences, 46 (1989) 3077-3107.

[46] M. Nakanishi and H. Niino, "An improved mellor-yamada level-3 model: Its numerical stability and application to a regional prediction of advection fog", Boundary-Layer Meteorology, 119 (2006) 397-407.

[47] M. Ek, K. Mitchell, Y. Lin, E. Rogers, P. Grunmann, V. Koren, G. Gayno, and J. Tarpley, "Implementation of noah land surface model advances in the national centers for environmental prediction operational mesoscale eta model", Journal of Geophysical Research: Atmospheres, 108 (2003).

[48] "Geoportale della regione basilicata". http://rsdi.regione. basilicata.it/Catalogo/srv/ita/search/?uuid=r_basili: 9EE9C09A-7091-D8A2-A41D-F28191B86C4F

[49] T.C. Project, CGAL: User and Reference Manual. CGAL Editorial Board, 5.0.2 edition, 2020.

[50] J.H. Ferziger, M. Perić, and R.L. Street, Computational methods for fluid dynamics, volume 3. Springer, 2002.

[51] "openfoam - buoyantboussinesqpisofoam". https://openfoamwiki. net/index.php/BuoyantBoussinesqPisoFoam. 
[52] O. Temel and J. van Beeck, "Adaptation of mesoscale turbulence parameterisation schemes as rans closures for abl simulations", Journal of Turbulence, 17 (2016) 966-997, doi: 10.1080/14685248.2016.1211286

[53] P. Taylor and H. Teunissen, "The askervein hill project: overview and background data", Boundary-layer meteorology, 39 (1987) 15-39.

[54] P. Taylor and H. Teunissen, "The askervein hill project: report on the september/october 1983 main field experiment", Atmospheric Environment Service, MSRB-84-6.(Downsview, ON), (1985). 